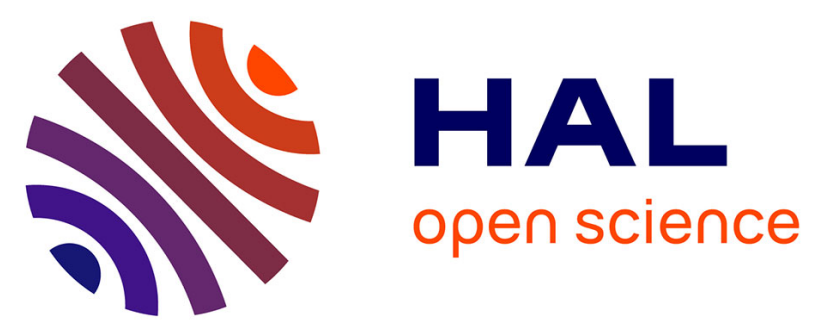

\title{
Modelling and numerical assessment of a maintenance strategy with stock through Piecewise Deterministic Markov Processes and Quasi Monte Carlo methods
} Jeanne Demgne, Sophie Mercier, William Lair, Jérôme Lonchampt

\section{- To cite this version:}

Jeanne Demgne, Sophie Mercier, William Lair, Jérôme Lonchampt. Modelling and numerical assessment of a maintenance strategy with stock through Piecewise Deterministic Markov Processes and Quasi Monte Carlo methods . Proceedings of the Institution of Mechanical Engineers, Part O: Journal of Risk and Reliability, 2017, 231 (4), pp.429-445. 10.1177/1748006X17712121 . hal-01576962

\section{HAL Id: hal-01576962 \\ https://hal.science/hal-01576962}

Submitted on 24 Aug 2017

HAL is a multi-disciplinary open access archive for the deposit and dissemination of scientific research documents, whether they are published or not. The documents may come from teaching and research institutions in France or abroad, or from public or private research centers.
L'archive ouverte pluridisciplinaire HAL, est destinée au dépôt et à la diffusion de documents scientifiques de niveau recherche, publiés ou non, émanant des établissements d'enseignement et de recherche français ou étrangers, des laboratoires publics ou privés. 


\title{
Modelling and numerical assessment of \\ a maintenance strategy with stock \\ through Piecewise Deterministic \\ Markov Processes and Quasi Monte \\ Carlo methods
}

\section{Jeanne Demgne ${ }^{1}$ and Sophie Mercier ${ }^{2}$ and William Lair ${ }^{3}$ and Jérôme Lonchampt ${ }^{1}$}

\begin{abstract}
To ensure a power generation level, the French national electricity supply (EDF) has to manage its producing assets by putting in place adapted preventive maintenance strategies. In this paper, a fleet of identical components is considered, which are spread out all around France (one per power plant site). The components are assumed to have stochastically independent lifetimes but they are made functionally dependent through the sharing of a common stock of spare parts. When available, these spare parts are used for both corrective and preventive replacements, with priority to corrective replacements. When the stock is empty, replacements are delayed until the arrival of new spare parts. These spare parts are expensive and their manufacturing time is long, which makes it necessary to rigorously define their ordering process. The point of the paper is to provide the decision maker with the tools to take the right decision (make or not the overhaul). To do that, two indicators are proposed, which are based on an economic variable called the Net Present Value (NPV). The NPV stands for the difference between the cumulated discounted cash-flows of the purely corrective policy and the one including the overhaul. Piecewise Deterministic Markov Processes (PDMPs) are first considered for the joint modelling of the stochastic evolution of the components, stock and ordering process with and without overhaul. The indicators are next expressed with respect to these PDMPs, which have to be numerically assessed. Instead of using the most classical Monte Carlo (MC) simulations, we here suggest alternate methods based on quasi Monte Carlo simulations, which replace the random uniform numbers of the MC method by deterministic sequences called Low Discrepancy Sequences. The obtained results show a real gain of the quasi Monte Carlo methods in comparison with the MC method. The developed tools can hence help the decision maker to take the right decision.
\end{abstract}

\section{Keywords}

Reliability, Maintenance strategy, Quasi Monte Carlo methods, Markov chain simulation, Net Present Value, Piecewise Deterministic Markov Process

\section{Introduction}

One major concern in reliability is the study of preventive maintenance policies, which aims at enlarging systems lifetimes and/or reducing their operating costs. A case study is here proposed: A fleet of identical components is considered, which are spread out all around France (one per power plant site). Based on their different locations, the components are not structured in any kind of system. Also, their lifetimes can be considered as independent random variables. The components are however made functionally dependent through the sharing of a common stock of spare parts.
These spare parts are expensive and their manufacturing time is long. Also, lack of spare parts entails some delay in the replacement of down components and some high unavailability costs due to the induced shutdown

\footnotetext{
${ }^{1}$ EDF Lab Saclay - Industrial Risk Management, France ${ }^{2}$ Laboratoire de Mathématiques et de leurs Applications (UMR CNRS 5142) - Université de Pau et des Pays de l'Adour, France ${ }^{3}$ EDF Lab Saclay - Electric Grid Measurements and Information System, France

Corresponding author:

Jeanne Demgne, EDF Lab Saclay - Industrial Risk Management , 7 Boulevard Gaspard Monge, O3EF15, 91120 Palaiseau, France.

Email: jeanne.demgne@gmail.com
} 
of some part of the power plant. All these features make it necessary to rigorously define the ordering process of spare parts, in order to find a balance between the ordering of eventually useless spare parts and unavailability costs by lack of spare parts.

The purchasing price of spare parts and the high unavailability costs make it necessary to consider an alternative maintenance strategy than a purely corrective one in order to prevent failures and reduce unavailability periods. A general overhaul is then considered, which requires a still more rigorous ordering process and investments planning (details further). The point of the paper is to provide the decision maker with the tools to help him deciding whether this overhaul should be undertaken or not.

An important question now is: which kind of indicators will best help the decision maker to evaluate this putative investments plan and take the right decision? Several financial indicators can be considered. The most common indicator from the reliability literature is the expected cumulated $\operatorname{cost}^{7}$. However, this indicator only reflects some mean behaviour of the random cash-flow under a given investments plan, and does not provide any insight into its economical venture. Based on this, we propose to use other indicators based on the Net Present Value (NPV), which stands for the difference between the cumulated discounted cashflows of both current and new investments plans. In our context, the current investments plan corresponds to a purely corrective replacement strategy (no investments planning) whereas the new investments plan involves preventive replacement. Two quantities linked to the NPV are considered: firstly, the expected NPV, which simply stands for the difference between the respective mean discounted cash-flows of both current and new investments plans; secondly, the probability for the NPV to be negative, which represents the probability to regret the new investments plan and helps to better capture the economic venture possibly entailed by the new plan. Note that the cash-flows of both current and new investments plans share a common history up to the first time when they diverge and are hence dependent random variables. The assessment of the probability for the NPV to be negative requires to take into account this dependence, which makes the study more complex.

Though standard in economical literature ${ }^{1 ; 2}$, the NPV seems to be somewhat less common in reliability literature. There however are a few notable exceptions. For instance, some authors suggest to consider the reliability of a system in terms of its net present value, which corresponds to the difference between its "revenue generation capability" and "the cost of obtaining it" ${ }^{21}$. They next characterize the best level of reliability which maximizes the profit. Another paper focuses on the analysis of the life-cycle costs of a pump ${ }^{4}$, which are all summarized through their net present values. The authors next use these different NPVs for helping to choose the most cost-effective combination from the point of view of design, development, production and maintenance. Another study is devoted to the NPV (or "financial worth") of a maintenance policy for a multi-states deterioration system ${ }^{18}$, where the NPV corresponds to the generated revenues resulting from an increase flux service minus the cost-flows of the maintenance.

Conversely to the present study, the authors of the previous papers seem to only consider the expected $\mathrm{NPV}$, which does not require to take into account the dependence between the two considered cash-flows for its assessment. Also, the downtimes are assumed to be negligible and spare parts always available. This allow the authors to envision more intricate preventive maintenance policies than in the present paper, where all these difficult features are taken into account (together with priority to corrective replacements with respect to preventive ones and eventually postponed preventive replacements).

In view of a precise definition of the NPV, the random evolution of components, level of the spare parts stock and ordering process must be jointly modelled. This involves both continuous and discrete random variables and Piecewise Deterministic Markov Processes (PDMPs) are consequently well adapted ${ }^{6}$. To be more specific, the NPV requires the introduction of two stochastically dependent PDMPs for its definition, for modelling both the current and new investments plans. Once both maintenance strategies are modelled, the decision criteria are next expressed with respect to the two PDMPs. The numerical evaluation of the decision criteria hence requires the numerical assessment of the distribution of the two dependent PDMPs. Several methods have been proposed in the literature for the numerical assessment of PDMPs, among which the most classical Monte Carlo (MC) simulations ${ }^{22}$. The main drawback of MC method is its slow convergence, which may entail long computational times. PDMPs may also be quantified by finite volume schemes ${ }^{10 ; 13}$. These methods are however not adapted to the 
assessments of PDMPs for which the dimension of the continuous part is greater than five. As the referred industrial case requires a higher dimension, they do not seem appropriate. Quantization methods ${ }^{3 ; 20}$ are also possible. However, they require the preliminary and computationally expensive construction of an "optimal" discrete grid, which synthesizes the state space of the PDMP. In the present study, the dimension of the state space evolves over time due to the ordering process and arrival of spare parts. The quantization method would require the construction of all optimal grids for all possible dimensions and it does not seem to be adapted to our context either.

Based on these considerations, it seems that only MC methods are available from the previous literature to assess the distribution of the NPV. We here propose to use Quasi Monte Carlo (QMC) methods as an alternative, which are well-known for often having a faster convergence than crude MC method. Let us recall that QMC methods have first been developed in the context of numerical integration, where they substitute the random uniform variables of the MC method by deterministic sequences with better uniformity properties, the so-called Low Discrepancy Sequences $(\mathrm{LDS})^{19}$. Following previous studies ${ }^{12}$, a specific LDS is used here: the Sobol sequence. A first possibility to use QMC method in our context is to interpret the quantities of interest (expected NPV and probability for the NPV to be negative) as an expectation of a functional of PDMPs (or of the underlying discrete time Markov chains, equivalently), and hence boil down to some classical numerical integration problem. Based on the underlying Markov chain structure of a PDMP, another possibility is to use a specific QMC method devoted to the assessment of Markov chains $8 ; 14$. This method consists in simulating several copies of Markov chains in parallel and in reordering copies at each step in ascending order. The sorting step allows some mixing between the copies and provides a better estimation of the distribution of the Markov chain ${ }^{16}$. A randomized version of this method is called Array Randomized Quasi Monte Carlo method in the literature ${ }^{16}$. This method is hence called Array Quasi Monte Carlo (AQMC) method here after. Note that the previous works on (A)QMC methods were devoted to the quantification of one single Markov chain with a simpler transition kernel than in the present paper. We hence need to face two additional difficulties, one induced by the complexity of the
Markov transition kernel of each of the two involved PDMPs, the other by the dependence between the two PDMPs. This requires some subtle adaptation of the previously developed (A)QMC methods (including a rigorous use of the elements of LDS). Randomized versions of (A)QMC methods are also considered later on, which consist in replacing the deterministic LDS of the (A)QMC methods by some randomized version. These are called Randomized (Array) Quasi Monte Carlo methods hereafter.

The structure of the article is as follows: the first section introduces both corrective and preventive maintenance strategies, as well as the NPV. Then, the second section presents the joint modelling of the components and stock under the two maintenance strategies through PDMPs. The numerical assessment of the NPV by MC, (A)QMC and randomized (A)QMC methods are presented in the third section. Numerical results are provided in the fourth section and concluding remarks end the paper.

\section{Presentation of the problem}

A fleet of $n$ identical and stochastically independent components is considered, which share a common stock of spare parts. These components are subject to corrective and preventive replacements (CR and PR). Though other preventive maintenance (PM) actions are performed in reality (such as adjustments, cleaning, ...), we here consider that the effects of such PM actions are taken into account in parameters of the probability distribution of components lifetime. Hence, only replacements are envisioned as possible maintenance actions in all the following. At initial time, the stock is assumed to contain $S$ spare parts and the supply time is deterministic, denoted by $\tau$. Moreover, the components of the fleet are assumed to be new at time $t=0$. The corrective and preventive maintenance strategies are compared through the NPV on a fixed operation horizon term denoted by $H$. Both strategies are now presented.

\section{The corrective maintenance policy}

The corrective maintenance (CM) strategy consists in replacing a failed component by a new one if a spare part is available. If the stock is empty at a failure time, the failed component becomes unavailable and it remains down until a spare part becomes available. The ordering process of a new spare part is as follows: 
- On $[0, H-\tau)$, a new spare part is ordered at each failure of a component.

- On $[H-\tau, H]$, no order is made. Indeed, the spare part would be delivered after the operation horizon term.

\section{The preventive maintenance policy}

The preventive replacement (PR) is an exceptional maintenance action. It takes place only once on the operation term, at some time $\zeta$, fixed in advance (with $\zeta<H-\tau)$. The PM strategy consists in preventively replacing at time $\zeta$, all components which did not fail before. With that aim, spare parts are ordered at time $\zeta-\tau$. Note that in the preventive strategy, the stock of spare parts is common to both $\mathrm{CR}$ and PR. A CR has a priority on a preventive one in the sense that, if CR are pending at time $\zeta$, they are first carried out. After all pending $\mathrm{CR}$ have been performed at time $\zeta$, PM actions take place if remaining spare parts are still available. When only a part of components awaiting a PR can be replaced (by lack of spare parts), the components to be replaced are randomly selected among all the equally likely components awaiting a PR. In this case, the PR of the remaining components is deferred until a new spare part becomes available.

The ordering process of a new spare part is the following:

- On $[0, \zeta-\tau)$, a new spare part is ordered at each failure of a component.

- At time $\zeta-\tau$, if there are components that have never failed before, spare parts are ordered in readiness for their $\mathrm{PR}$ at time $\zeta$.

- On $(\zeta-\tau, \zeta)$, a spare part is ordered at failure of a component $\mathrm{C}$ (say) but only in the case where component $\mathrm{C}$ has already suffered a failure before. Indeed, in the opposite case, the PR of component $\mathrm{C}$ has been planned at time $\zeta$ and a spare part has already been ordered for its replacement. This spare part will be used for the $\mathrm{CR}$ of component $\mathrm{C}$ at $\zeta$.

- At time $\zeta$, spare parts arrive, which are used first for CR and next for PR. Some of these replacements may be deferred by lack of enough available spare parts.

- On $(\zeta, H-\tau)$, a spare part is ordered at failure of a component $\mathrm{C}$ which has already been replaced ( $\mathrm{CR}$ or PR). Indeed, in the other case, the PR of component $\mathrm{C}$ has been deferred at time $\zeta$ and an order is already in progress for its replacement.

- On $[H-\tau, H]$, no order is made.

\section{Costs data}

The cost function associated to an investments plan takes into account discounted costs due to CR or PR, purchase of spare parts and components unavailability. An exponential discounting is considered, where a cost $C$ at time $t$ corresponds to a discounted cost $C e^{-\alpha t}$ at time 0 , with $\alpha$ the discounted rate. The costs of corrective $\left(c_{c}\right)$ and preventive $\left(c_{p}\right)$ replacements are cashed at replacement times. They correspond to intervention costs and do not include the purchase cost of spare parts. In case of a planned replacement at time $\zeta$, the purchase cost of a spare part $\left(c_{s}^{p}\right)$ is cashed at delivery. In any other case (and any other time), it is immediately cashed when a spare part is ordered and is denoted by $c_{s}^{c}$. The unitary downtime cost per component and unit time is denoted by $C_{u n a}$. Downtime costs are cashed at the end of unavailability periods.

\section{The Net Present Value}

As mentioned previously, the NPV stands for the difference between the cumulated discounted cash-flows of both strategies ( $\mathrm{CM}$ and PM). The NPV is zero as long as the two strategies coincide. The time $T$ when the two strategies first differ depends on the scenario: if all components have already suffered a failure before $\zeta-\tau$, then no $\mathrm{PR}$ is planned at time $\zeta$, and $T$ is equal to $H(\mathrm{NPV}=0)$. On the contrary, if there is at least one component which has not suffered any failure up to time $\zeta-\tau$, then a spare part is ordered in view of its $\mathrm{PR}$ at time $\zeta$, and this makes the two $\mathrm{CM}$ and $\mathrm{PM}$ strategies to differ from time $T=\zeta-\tau$. Note that, even in that case, the two strategies coincide on $[0, \zeta-\tau)$ and the NPV is always zero on $[0, \zeta-\tau)$. After time $\zeta-\tau$, the two maintenance strategies are assumed to evolve independently. Note however that they are correlated through their common history up to time $\zeta-\tau$, which entails some dependence between the cumulated costs associated to each strategy. 


\section{Modelling through PDMPs}

\section{Description of the constitutive parts of the PDMPs}

Piecewise Deterministic Markov Processes (PDMPs) have been introduced by Davis M.H.A ${ }^{6}$ in a general setting. A PDMP is a hybrid process $\left(I_{t}, X_{t}\right)_{t \geq 0}$. The first part $I_{t}$ is discrete, with values in a discrete state space $E$. The second part $X_{t}$ is almost surely continuous and takes range in a Borel subset $B \subset \mathbb{R}^{d}$. It usually represents environmental conditions such as temperature or pressure, but it can also represent the time to the next failure or to the next arrival of a spare part, as in the present study. The process $\left(I_{t}, X_{t}\right)_{t>0}$ jumps at random isolated times. Between jumps, the discrete part $I_{t}$ is constant and the evolution of the (almost surely) continuous part $X_{t}$ is deterministic.

In our context, a PDMP is used to model the state (up/down) of the $n$ components, the times at which their states change, the size of the stock, the arrival times of the spare parts as well as the cost function under each maintenance strategy. This leads us to define the following variables as the constitutive parts of our PDMP:

- $X_{t}=\left(X_{1, t}, \ldots, X_{n, t}\right)$ where, for $1 \leq j \leq n$, the signification of $X_{j, t}$ differs according to whether the corresponding component is up or down. If it is up, $X_{j, t}$ is the time of its future failure $\left(X_{j, t}>t\right)$. If it is down, $X_{j, t}$ is its last time of failure $\left(X_{j, t} \leq t\right)$. Note that the place of $X_{j, t}$ with respect of $t$ indicates whether the component is up or down at time $t$ and that it always represents a failure time, either past or future.

- $I_{t}=\left(I_{1, t}, \ldots, I_{n, t}\right)$ is a failure indicator: for $1 \leq$ $j \leq n$, if the component corresponding to $X_{j, t}$ has never failed before $t$, then $I_{j, t}=1$, else $I_{j, t}=0$. Thus at time $t, K_{t}=\sum_{j=1}^{n} I_{j, t}$ represents the number of components that have never been replaced on $[0, t]$.

The vector $\left(X_{t}, I_{t}\right)=\left(\left(X_{1, t}, I_{1, t}\right), \ldots,\left(X_{n, t}, I_{n, t}\right)\right)$ is sorted in ascending order according to $X_{t}$. This entails that $\left(X_{j, t}, I_{j, t}\right)$ does not correspond to a given component and that the corresponding component will change over time. Note that the failure time and failure indicator are kept together in order to know which components remain to be preventively replaced.

- $S_{t}$ is a variable which is decreased by 1 at each failure time and increased by 1 at each delivery of one spare part. If $S_{t} \geq 0$, then $S_{t}$ represents the number of available spare parts at time $t$, else $-S_{t}$ is the number of down components at time $t$.

- $D_{t}=\left(D_{1, t}, \ldots, D_{m, t}\right)$ represent the predicted times for spare parts arrivals where $m$ is the number of orders in progress. If no order is in progress, $D_{t}=0$ (say).

- $L_{t}$ is the number of components at time $t$ awaiting a deferred PR.

- $C_{t}$ is the cumulated discounted cost at time $t$.

The process $Z=\left(Z_{t}\right)_{t \geq 0}=\left(Y_{t}, t\right)_{t \geq 0}=$ $\left(\left(X_{t}, I_{t}\right), S_{t}, D_{t}, L_{t}, C_{t}, t\right)_{t>0}$ is a PDMP whose discrete part is $\left(I_{t}, S_{t}, L_{t}\right)$ and continuous part is $\left(X_{t}, D_{t}, C_{t}, t\right)$. The jumps of $Z$ correspond either to the failure of one component, to the arrival of a spare part or to planned replacements at time $\zeta$ (case of the PM strategy). Based on the fact that costs are cashed at the end of unavailability periods (which correspond to spare part arrivals), they are hence always cashed at jump times (and also eventually at time $H$ ) and do not evolve between jumps. Then, looking at the other constitutive parts of the PDMP, it is clear that the only thing which evolves between jumps is the time $t$, so that $Y_{t}$ is constant between jumps. Setting $\left(T_{k}\right)_{k \geq 0}$ to be the jump times of $\left(Z_{t}\right)_{t \geq 0}$, simulating trajectories of the $\operatorname{PDMP}\left(Z_{t}\right)_{t \geq 0}$ is hence equivalent to simulating trajectories of the underlying Markov chain $\left(Z_{k}\right)_{k \geq 0}=\left(Y_{T_{k}}, T_{k}\right)_{k \geq 0}$. Both the PDMP $\left(Z_{t}\right)_{t \geq 0}$ and the Markov chain $\left(Z_{k}\right)_{k>0}$ are denoted by $Z$ in the following, where the distinction between the two will be clear from the context.

We make use of several PDMPs with similar constitutive parts in the sequel. To distinguish between them, we add a superscript $(i)$ to all of the previous notations (e.g. $\left.Z_{t}^{(i)}, T_{k}^{(i)}, \ldots\right)$.

\section{Modelling the corrective maintenance strategy}

The CM strategy is modelled with the $\operatorname{PDMP} Z^{(1)}=$ $\left(Z_{t}^{(1)}\right)_{t \geq 0}$. Though useless here, the variable $I_{t}^{(1)}$ is kept in $Z_{t}^{(1)}$ because it will be useful later on for initializing the PM strategy. The variable $K_{\zeta-\tau}^{(1)}=\sum_{j=1}^{n} I_{j, \zeta-\tau}^{(1)}$ stands for the number of spare parts to be ordered at time $\zeta-\tau$ in readiness for $\mathrm{PR}$ at time $\zeta$. Also, the variable $L_{t}^{(1)}$ in $Z_{t}^{(1)}$ is here always equal to 0 and hence useless. It is kept on only for sake of coherence of the notations. The Markov chain $Z^{(1)}=\left(Z_{k}^{(1)}\right)_{k \geq 0}$ is now 
defined as follows:

$$
\left\{\begin{array}{l}
Z_{0}^{(1)}=\left(\left(X_{0}^{(1)}, I_{0}^{(1)}\right), S_{0}^{(1)}, D_{0}^{(1)}, L_{0}^{(1)}, C_{0}^{(1)}, T_{0}^{(1)}\right) \\
Z_{k+1}^{(1)}=\phi_{1}\left(Z_{k}^{(1)}, U_{k+1}\right) ; U_{k+1} \sim \mathcal{U}([0,1]), k \geq 0
\end{array}\right.
$$

where, for any $d \in \mathbb{N}^{*}$, symbol $\mathcal{U}\left([0,1]^{d}\right)$ stands for the uniform distribution on $[0,1]^{d}$. The details for both the initialization and transition function $\phi_{1}$ are presented in Appendix A.

Setting $N_{H}^{(1)}$ to be the number of jumps of the PDMP $Z^{(1)}$ on $[0, H]$, the Markov chain $Z^{(1)}$ allows to model the evolution of the PDMP up to the time $T_{N_{H}^{(1)}}^{(1)}$ of the last jump of the PDMP on $[0, H]$. However, if a component is down at time $H$, its unavailability cost has not yet been taken into account at time $T_{N_{H}^{(1)}}^{(1)}$. In that case, the corresponding downtime has to be added from the failure time $X_{j, H}^{(1)}=X_{j, T_{N_{H}^{(1)}}^{(1)}}^{(1)}$ up to the horizon term $H$. This writes:

$$
\begin{aligned}
C_{H}^{(1)} & =C_{N_{H}^{(1)}}^{(1)}+C_{\text {una }} \sum_{j=1}^{n}\left(\int_{X_{j, H}^{(1)}}^{H} e^{-\alpha t} d t\right) \mathbf{1}_{\left\{X_{j, H}^{(1)} \leq H\right\}} \\
& =C_{N_{H}^{(1)}}^{(1)}+\frac{C_{u n a}}{\alpha} \sum_{j=1}^{n}\left(e^{-\alpha X_{j, H}^{(1)}}-e^{-\alpha H}\right) \mathbf{1}_{\left\{X_{j, H}^{(1)} \leq H\right\}}
\end{aligned}
$$

\section{Modelling the preventive maintenance strategy}

As presented before, the PM strategy consists in replacing at time $\zeta$ all components which have never been replaced before. Thus, spare parts are eventually ordered at time $\zeta-\tau$ in view of $\mathrm{PR}$ at time $\zeta$. In this case, the ordering process differs on $[0, \zeta-\tau),[\zeta-\tau, \zeta)$ and $[\zeta, H]$ so that three different PDMPs are necessary to model the PM strategy. We consequently set:

$$
Z_{t}^{(2)}=\left\{\begin{array}{l}
Z_{t}^{(1)} \quad \text { if } 0 \leq t<\zeta-\tau \\
Z_{t-\zeta+\tau}^{(3)} \quad \text { if } \zeta-\tau \leq t<\zeta \\
Z_{t-\zeta}^{(4)} \quad \text { if } \zeta \leq t \leq H
\end{array}\right.
$$

where $\left(Z_{t}^{(1)}\right)_{0 \leq t<\zeta-\tau}$ models the CM strategy on $[0, \zeta-\tau)$ and where $\left(Z_{t-\zeta+\tau}^{(3)}\right)_{\zeta-\tau \leq t<\zeta}$ and $\left(Z_{t-\zeta}^{(4)}\right)_{\zeta \leq t \leq H}$ model the evolution of the PM strategy on $[\zeta-\tau, \bar{\zeta})$ and $[\zeta, H]$, respectively. The details of the initialization and of the transition functions of the Markov chains $Z^{(3)}$ and $Z^{(4)}$ are provided in Appendices B and C, respectively.

Note that in case all components have failed before time $\zeta-\tau$, both $\mathrm{CM}$ and $\mathrm{PM}$ strategies coincide so that $Z_{t}^{(2)}=Z_{t}^{(1)}, \forall t \geq 0$. Also, just as for the CM strategy, the downtime cost of down components at time $H$ is cashed at the operation horizon term $H$.

\section{The Net Present Value}

Remembering that the NPV stands for the difference between the cumulated discounted costs of both CM and PM strategies, the NPV up to horizon $H$ is:

$$
\begin{aligned}
& N P V(H) \\
& =N P V([0, H]) \\
& =N P V([\zeta-\tau, H]) \mathbf{1}_{\left\{K_{\zeta-\tau}^{(1)}>0\right\}} \\
& =\left(C^{(1)}([\zeta-\tau, H])-C^{(2)}([\zeta-\tau, H])\right) \mathbf{1}_{\left\{K_{\zeta-\tau}^{(1)}>0\right\}}
\end{aligned}
$$

where $C^{(1)}([\zeta-\tau, H])$ and $C^{(2)}([\zeta-\tau, H])$ are dependent through the state of components and stock at time $\zeta-\tau$, and where we recall that $K_{\zeta-\tau}^{(1)}$ stands for the number of spare parts to be ordered at time $\zeta-\tau$ in readiness for PR at time $\zeta$ (for strategy 1 ).

The quantities of interest are $\mathbb{E}[N P V(H)]$ and $\mathbb{P}(N P V(H) \leq 0)$. These quantities depend on all events which occur during $[0, H]$ in both strategies. They can be expressed as $\mathbb{E}\left[\Phi\left(\left(Z_{k_{1}}^{(1)}, Z_{k_{2}}^{(2)}\right)_{0 \leq k_{1} \leq N_{H}^{(1)}, 0 \leq k_{2} \leq N_{H-\zeta+\tau}^{(2)}}\right)\right] \quad$ where $N_{H-\zeta+\tau}^{(2)}$ stands for the number of jumps of $Z^{(2)}$ on $[\zeta-\tau, H]$ and where $\Phi$ is a complicate and non explicit function.

Both maintenance strategies are now modelled, the next step is the evaluation of the chosen criteria. The proposed methods are presented in the next section.

\section{Numerical assessment of the Net Present Value \\ Monte Carlo method}

Let us first note that the two Net Present Value indicators of interest may be written under the shape $\mathbb{E}[h(N P V(H))]$, where $h(x)=x$ for the expected $N P V(H)$ and $h(x)=\mathbf{1}_{\{x \leq 0\}}$ for the probability for the $N P V$ to be negative at time $H$. As mentioned in the introduction, MC simulations are classically used to evaluate such quantities (or any other quantity of the shape $\mathbb{E}[h(N P V(H))])$. In our case, sample paths of each Markov chain $\left(Z_{k}^{(i)}\right)_{k \geq 0}, i=\{1,3,4\}$, are sequentially simulated using its initialization and 
transition function. Samples of $N P V(H)$ (and next of $h(N P V(H)))$ are derived using Equation (3). The empirical mean of the $h(N P V(H))$ sample provides an estimation of the corresponding theoretical quantity. Confidence intervals are given by the central limit theorem.

\section{Quasi Monte Carlo method}

Quasi Monte Carlo for general Markov chains. We first consider a general Markov chain $Z=\left(Z_{k}\right)_{k \geq 0}$ which takes range in $E \subseteq \mathbb{R}^{s}$ and such that

$$
\left\{\begin{array}{l}
Z_{0}=z_{0} \in E \\
Z_{k+1}=\varphi_{k}\left(Z_{k}, U_{k+1}\right) ; U_{k+1} \sim \mathcal{U}\left([0,1]^{d}\right), k \geq 0 .
\end{array}\right.
$$

The point is to see how to simulate $N$ realizations of $\left(Z_{1}, \ldots, Z_{m}\right)$ by QMC method, with $m \in \mathbb{N}^{*}$ fixed. Let us first note that, due to the recursive construction, $Z_{k}$ can be written as

$$
Z_{k}=\Phi_{k}\left(z_{0},\left(U_{1}, \ldots, U_{k}\right)\right)=\Phi_{k}\left(z_{0}, \mathbf{U}_{k}\right)
$$

where $\mathbf{U}_{k}=\left(U_{1}, \ldots, U_{k}\right) \sim \mathcal{U}\left([0,1]^{k \times d}\right)$ for all $1 \leq k \leq$ $m$. The simulation of one realization of $\left(Z_{1}, \ldots, Z_{m}\right)$ hence requires a random uniform variable $\mathbf{U}_{m}$ with dimension $m \times d$, where the $k \times d$ first dimensions $\left(\mathbf{U}_{k}\right)$ are used for $Z_{k}$. As mentioned in the introduction, the QMC method consists in replacing the random uniform variable by a LDS with the same dimension. The random uniform variable $\mathbf{U}_{m}$ is hence replaced by a LDS $\overline{\mathbf{U}}^{m \times d}$ with dimension $m \times d$. To simulate $N$ realizations $\left(z_{1}^{i}, \ldots, z_{m}^{i}\right)_{1 \leq i \leq N}$ of $\left(Z_{1}, \ldots, Z_{m}\right)$, the $N$ first elements $\left(\overline{\mathbf{u}}_{i}^{m \times d}\right)_{1<i<N}$ of the LDS $\overline{\mathbf{U}}^{m \times d}$ are used, with $\overline{\mathbf{u}}_{i}^{m \times d}=\left(\bar{u}_{i}^{1}, \ldots, \bar{u}_{i}^{m \times d}\right)$. For each $1 \leq i \leq N$ and each $1 \leq k \leq m$, we use $d$ successive coordinates of $\overline{\mathbf{u}}_{i}^{m \times d}$ to generate the $i$-th realization $z_{k}^{i}$ of $Z_{k}$ from $z_{k-1}^{i}$ with

$$
\begin{aligned}
z_{k}^{i} & =\varphi_{k}\left(z_{k-1}^{i},\left(\bar{u}_{i}^{(k-1) \times d+1}, \ldots, \bar{u}_{i}^{k \times d}\right)\right) \\
& =\Phi_{k}\left(z_{0}^{i},\left(\bar{u}_{i}^{1}, \ldots, \bar{u}_{i}^{k \times d}\right)\right) .
\end{aligned}
$$

In that way, one element $\overline{\mathbf{u}}_{i}^{m \times d}$ is used to assess one realization $\left(z_{1}^{i}, \ldots, z_{m}^{i}\right)$. In the general case where the function $\Phi_{k}$ is not explicit (as in the present paper), the generation of the $i$-th realization of $\left(Z_{1}, \ldots, Z_{m}\right)$ is made sequentially through (4), as in the MC method.

As can be seen, the dimension of the LDS used for simulating $N$ realizations of $\left(Z_{1}, \ldots, Z_{m}\right)$ depends on $m$, which should consequently be fixed in advance. When the number of steps to be simulated (say $M$ ) is random, one must fix in advance the maximal number of possible steps. A possibility is to chose $m$ such that $\mathbb{P}(M>m)$ is very small and consider a LDS with dimension $m \times d$.

Quasi Monte Carlo method for the Net Present Value. Based on Subsection "The Net Present Value", one can see that the NPV depends on $N_{H}=N_{H}^{(1)}+N_{H-\zeta+\tau}^{(2)}$ uniform random variables, where $N_{H}^{(1)}$ and $N_{H-\zeta+\tau}^{(2)}$ refer to $\mathrm{PDMP} Z^{(1)}$ and $Z^{(2)}$, respectively. In order to fix the maximal number of possible jumps (see the end of the previous subsection), one may consider two possibilities:

- Chose $m^{(1)}$ and $m^{(2)}$ such that both $\mathbb{P}\left(m^{(1)}<N_{H}^{(1)}\right)$ and $\mathbb{P}\left(m^{(2)}<N_{H-\zeta+\tau}^{(2)}\right)$ are very small and build a $\left(m^{(1)}+m^{(2)}\right)$-dimensional LDS. In that case, the idea is to use the $m^{(1)}$ first dimension terms of the LDS for simulating $Z^{(1)}$ and the $m^{(2)}$ last dimension terms for $Z^{(2)}$.

- Chose $m$ such that $\mathbb{P}\left(m<N_{H}\right)$ is very small and construct a LDS with dimension $m$. The successive jumps of $Z^{(1)}$ and next of $Z^{(2)}$ are assessed using successive dimensions of the LDS.

The discrepancy of a LDS is known to increase when the dimension increases ${ }^{5}$. As the first possibility will generally use higher dimensions terms of the LDS than the second, we prefer use the second method. Let $\overline{\mathbf{U}}^{m}=\left(\bar{U}^{k}\right)_{1<k<m}$ be a $m$-dimensional LDS and $\overline{\mathbf{U}}^{k: k+1}=\left(\bar{U}^{k}, \bar{U}^{k+1}\right)$, where $\bar{U}^{k}$ represents the $k$-th dimension of $\overline{\mathbf{U}}^{m}$. Just as for general Markov chains, the $N$ first elements of the LDS $\left(\overline{\mathbf{U}}_{i}^{m}\right)_{1 \leq i \leq N}$ are used to assess $\mathrm{N}$ realizations of the $N P V(H)$ (and next of $h(N P V(H)))$. One realization of the $N P V(H)$ (or of $Z_{k}^{(1)}, Z_{k}^{(3)}, Z_{k}^{(4)}$, equivalently) hence corresponds to one element of the LDS. The quantities of interest are estimated by the empirical mean of $h(N P V(H))$. Specific details concerning the numerical assessment of $N P V(H)$ using the QMC method are given in Algorithm 1. Note that this algorithm is just the same as the MC one, with uniform random variables substituted by elements of the LDS.

When the number $n$ of components increases, the required dimension for the LDS becomes larger, which may entail inaccuracy ${ }^{5}$. To overcome this, we propose to use Array Quasi Monte Carlo (AQMC) method, specially developed for the simulation of Markov chains. 


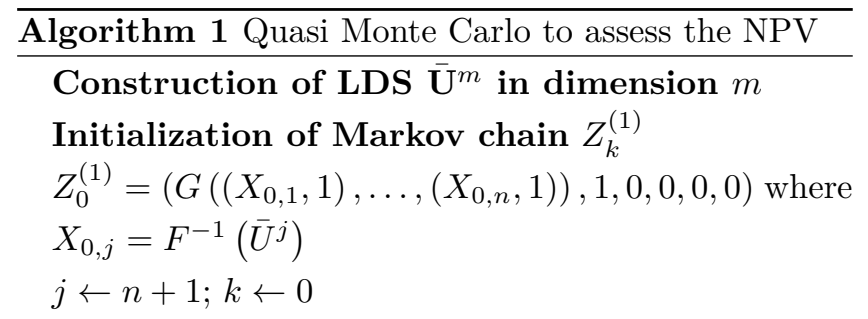

Simulation of CM strategy up to $\zeta-\tau$ to order spare parts in readiness for $\mathbf{P R}$

while $T_{k}^{(1)} \leq \zeta-\tau$ do

$$
Z_{k+1}^{(1)}=\phi_{1}\left(Z_{k}^{(1)}, \bar{U}^{j}\right) ; j \leftarrow j+1 ; k \leftarrow k+1
$$

end while

Calculation of number of components which have never failed before $\zeta-\tau$

if $K_{\zeta-\tau}^{(1)}=0$ then both strategies are identical and the NPV at time $H$ equals 0

else initialization of PM strategy: $Z_{0}^{(3)}$

end if

Simulation of CM strategy up to horizon time while $T_{k}^{(1)} \leq H$ do

$$
Z_{k+1}^{(1)}=\phi_{1}\left(Z_{k}^{(1)}, \bar{U}^{j}\right) ; j \leftarrow j+1 ; k \leftarrow k+1
$$

end while

$$
\begin{aligned}
C_{H}^{(1)} & =C_{N_{H}^{(1)}}^{(1)} \\
& +\frac{C_{\text {una }}}{\alpha} \sum_{j=1}^{n}\left(e^{-\alpha X_{j, N_{H}^{(1)}}^{(1)}}-e^{-\alpha H}\right) \mathbf{1}_{\left\{X_{j, N_{H}^{(1)}}^{(1)} \leq H\right\}}
\end{aligned}
$$

if $K_{\zeta-\tau}^{(1)}>0$ then

Simulation of PM strategy up to time $\zeta$

$k \leftarrow 0$

$$
\begin{aligned}
& \text { while } T_{k}^{(3)} \leq \zeta \text { do } \\
& \qquad \begin{array}{l}
Z_{k+1}^{(3)}=\phi_{3}\left(Z_{k}^{(3)}, \bar{U}^{j}\right) \\
j \leftarrow j+1 ; k \leftarrow k+1
\end{array}
\end{aligned}
$$

end while

Preventive replacements at time $\zeta$

The initialization of $Z_{0}^{(4)}$ depends on the number of unavailable components at time $\zeta$. Denote by $N_{\zeta}$ the number of random variables needed for initializing $Z_{0}^{(4)}$. Then, the $N_{\zeta}$ following components of $\overline{\mathbf{U}}^{m}$ are used to initialize $Z_{k}^{(4)}$.

$$
j \leftarrow j+N_{\zeta} ; k \leftarrow 0
$$

Simulation of PM strategy up to horizon time $H$

$$
\begin{aligned}
& \text { while } T_{k}^{(4)} \leq H \text { do } \\
& \qquad \begin{array}{l}
\left.Z_{k+1}^{(4)}=\phi_{4}\left(Z_{k}^{(4)}, \overline{\mathbf{U}}^{j: j+1}\right)\right) \\
j \leftarrow j+1 ; k \leftarrow k+1
\end{array}
\end{aligned}
$$

end while

$$
\begin{aligned}
& C_{H}^{(2)}=C_{N_{H-\zeta}^{(4)}}^{(4)}+ \\
& \frac{C_{u n a}}{\alpha} \sum_{j=1}^{n}\left(e^{-\alpha X_{j, N_{H-\zeta}^{(4)}}^{(4)}-e^{-\alpha H}}\right) \mathbf{1}_{\left\{X_{j, N_{H-\zeta}^{(4)}}^{(4)} \leq H\right\}} \leq
\end{aligned}
$$

else $C_{H}^{(2)}=C_{H}^{(1)}$

end if

return $N P V(H)=C_{H}^{(1)}-C_{H}^{(2)}$

\section{Array Quasi Monte Carlo method}

Array Quasi Monte Carlo for general Markov chains. Let us start again from a general Markov chain $\left(Z_{k}\right)_{k \geq 0}$ as in Subsection "Quasi Monte Carlo for general Markov chains", where we recall that a $d$-dimensional uniform random variable is required at each step. Here, the chain is assumed to take range in $\mathbb{R}$ for sake of simplicity $(s=1)$. The principle of AQMC method is to simulate $N$ trajectories of $\left(Z_{1}, \ldots, Z_{m}\right)$ in parallel, with some mixing between them at each step. More specifically, a LDS $\mathbf{S}^{d+1}$ in dimension $d+1$ is considered, whose successive elements are denoted by $\mathbf{s}_{1}^{d+1}, \mathbf{s}_{2}^{d+1}, \ldots, \mathbf{s}_{p}^{d+1}, \ldots$ To simulate $N$ realizations of $\left(Z_{1}, \ldots, Z_{m}\right)$, the $N \times m$ first elements of $\mathbf{S}^{d+1}$ are used: $\left(\mathbf{s}_{1}^{d+1}, \ldots, \mathbf{s}_{N}^{d+1}\right)$ is used to simulate $N$ realizations of $Z_{1}$, next $\left(\mathbf{s}_{N+1}^{d+1}, \ldots, \mathbf{s}_{2 N}^{d+1}\right)$ is used for $Z_{2}$ and more generally, $\left(\mathbf{s}_{(k-1) N+1}^{d+1}, \ldots, \mathbf{s}_{k N}^{d+1}\right)$ is used for $Z_{k}($ all $1 \leq k \leq m)$. The mixing of the trajectories is made through a sorting step $^{8 ; 14}$, which we now describe specifically. Assume the $k$-th step of the $N$ chains in parallel is constructed (say $\left.\left(z_{k}^{i}\right)_{1 \leq i \leq N}\right)$, the $(k+1)$-th step is assessed as follows:

1. Relabel the chains $\left(z_{k}^{i}\right)_{1 \leq i \leq N}$ according to the first coordinate of each element of $\left(\mathbf{s}_{k N+1}^{d+1}, \ldots, \mathbf{s}_{(k+1) N}^{d+1}\right)$

2. Determine the next step of the $N$ chains using the $d$ remaining coordinates in each element of $\left(\mathbf{s}_{k N+1}^{d+1}, \ldots, \mathbf{s}_{(k+1) N}^{d+1}\right)$;

3. Sort the chains in ascending order: $z_{k+1}^{1} \leq \cdots \leq$ $z_{k+1}^{N}$.

This procedure is repeated until the desired number of steps is reached. When the chain takes range in $\mathbb{R}^{s}$ with $s>1$, a difficulty arises for the choice of the sorting function. A first possibility is to define a unidimensional sorting function, which may depend on all constitutive parts of the Markov chain ${ }^{16}$. In that case, a $(d+1)$-dimensional LDS is used as in the case $s=1$. Another mapping scheme is proposed in the literature ${ }^{8}$, 
which requires a $(d+s)$-dimensional LDS: the $s$ first dimensions are used to relabel the chains and the $d$ last ones to assess the next step. Both methods have been tested here with no evidence for one or the other method. We consequently consider a uni-dimensional function, which is simpler. Note that the efficiency of the AQMC method highly depends on a judicious choice of the sorting method and that a bad choice can even provide wrong estimations.

Whatever the sorting method is, the dimension of the $\operatorname{LDS}(d+1$ or $d+s)$ is not linked any more to the number of steps $(m)$, as in the QMC method. In the present study, the number of jumps of the PDMPs on $[0, H]$ increases significantly with the number of components. In case of numerous components, the AQMC method hence allows to radically reduce the dimension of the LDS when compared to the QMC method.

Array Quasi Monte Carlo for the Net Present Value. The assessment of $N P V(H)$ though the general AQMC method described in the previous subsection requires some further precision and specific non trivial adjustments. Indeed, the $N P V(H)$ is a function of two dependent Markov chains, which model the two maintenance strategies. This raises several questions:

- How to sort the Markov chains?

- How to keep the dependence between Markov chains which share the same history?

- What should be the dimension of the used LDS?

As seen before, the sorting function has an effect on the efficiency of the AQMC method. Some multivariate sorting function has been proposed in the literature ${ }^{15}$, where the Markov chains are successively sorted according to their different coordinates and aggregated into a certain number of packets at each sorting step. The main difficulty of this multivariate sort is to determine the numbers of packets to use at each sorting step. We have tested this method in our context but the successive sorting steps lead to very long computational times, so that it does not seem adapted. We have also tried to sort the chains according to their respective cumulated discounted costs and according to their next future jump times. The conclusion of those trials was that the most efficient method was to sort the chains in ascending order according to their next future jump times, which is done in the remaining of the paper.

The point now is to see how to deal with the dependence between the two dependent Markov chains
$Z^{(1)}$ and $Z^{(2)}$ in the AQMC method. During the sorting step of the parallel Markov chains, we have to keep the dependence between the copies of $Z^{(1)}$ and $Z^{(2)}$ which share the same history. With that aim, a vector which couples the chains with a common history is considered.

Finally, the dimension of the LDS to be used should be specified. As for the initialization step, a $n$-dimensional uniform random variable is required to predict the first failure times of all $n$ components and hence, a $(n+1)$ dimensional LDS is used. As for the transitions, one single lifetime is drawn at each failure time (if a spare part is available), which requires one single uniform random variable. Moreover, in the PM strategy, an additional uniform random variable may be necessary in case of a postponed PR, for the eventual choice of the component to be preventively replaced. Based on this analysis, an at most two-dimensional uniform random variable is required and a 3 -dimensional LDS is used.

An algorithm for the evaluation of the NPV using AQMC method is now presented. The following notations are considered:

- $N$ the number of copies in parallel;

- $\mathbf{S}^{l}$ : a LDS in dimension $l$ whose successive elements are $\mathbf{s}_{1}^{l}, \mathbf{s}_{2}^{l}, \ldots$;

- $\mathbf{s}_{i_{1}: i_{2}}^{l}=\left(\mathbf{s}_{i_{1}}^{l}, \mathbf{s}_{i_{1}+1}^{l}, \ldots, \mathbf{s}_{i_{2}}^{l}\right)$

- $\mathbf{s}_{i}^{l}(j)$ : the $j$-th dimension of element $\mathbf{s}_{i}^{l}$

- $\mathbf{s}_{i}^{l}\left(j_{1}: j_{2}\right)=\left(\mathbf{s}_{i}^{l}\left(j_{1}\right), \mathbf{s}_{i}^{l}\left(j_{1}+1\right), \ldots, \mathbf{s}_{i}^{l}\left(j_{2}\right)\right)$

- $\mathbf{s}_{i_{1}: i_{2}}^{l}\left(j_{1}: j_{2}\right)=\left(\mathbf{s}_{i_{1}}^{l}\left(j_{1}: j_{2}\right), \ldots, \mathbf{s}_{i_{2}}^{l}\left(j_{1}: j_{2}\right)\right)$

- $\mathbf{Z}_{k}^{(i)}=\left(Z_{k, 1}^{(i)}, Z_{k, 2}^{(i)}, \ldots, Z_{k, N}^{(i)}\right)$ : states of $N$ copies in parallel for Markov chain $\left(Z_{k}^{(i)}\right)_{k \geq 0}$;

- $\mathbf{Z}_{k}^{(i)}(1: M)=\left(Z_{k, 1}^{(i)}, Z_{k, 2}^{(i)}, \ldots, Z_{k, M}^{(i)}\right)$ the $M$ first copies of $\mathbf{Z}_{k}^{(i)}$;

- $\mathbf{Z}_{k+1}^{(i)}(1: M)=\phi_{i}\left(\mathbf{Z}_{k}^{(i)}(1: M), \mathbf{s}_{m: m+M-1}^{2}(1: j)\right)$ means that, $Z_{k+1, p}^{(i)}=\phi_{i}\left(Z_{k, p}^{(i)}, \mathbf{s}_{m+p-1}^{2}(1: j)\right)$, for $j \in\{1,2\}$ and $1 \leq p \leq M$;

- $\mathbf{Z}_{(k)}^{(i)}=\left(Z_{k,(1)}^{(i)}, Z_{k,(2)}^{(i)}, \ldots, Z_{k,(N)}^{(i)}\right)$ : elements of $\mathbf{Z}_{k}^{(i)}$ sorted in ascending order according to future jump times, namely $Z_{k,\left(p_{1}\right)}^{(i)} \leq Z_{k,\left(p_{2}\right)}^{(i)}$ if $T_{k,\left(p_{1}\right)}^{(i)} \leq T_{k,\left(p_{2}\right)}^{(i)} \forall p_{1}, p_{2}$;

- $\mathbf{Z}_{k_{1}, k_{2}}^{\left(i_{1}, i_{2}\right)}=\left(\mathbf{Z}_{k_{1}}^{\left(i_{1}\right)}, \mathbf{Z}_{k_{2}}^{\left(i_{2}\right)}\right)=$ $\left(\left(Z_{k_{1}, 1}^{\left(i_{1}\right)}, Z_{k_{2}, 1}^{\left(i_{2}\right)}\right), \ldots,\left(Z_{k_{1}, N}^{\left(i_{1}\right)}, Z_{k_{2}, N}^{\left(i_{2}\right)}\right)\right)$ the coupled chain of $\mathbf{Z}_{k_{1}}^{\left(i_{1}\right)}$ and $\mathbf{Z}_{k_{2}}^{\left(i_{2}\right)}$;

- $\mathbf{Z}_{\left(k_{1}\right), k_{2}}^{\left(i_{1}, i_{2}\right)}=\left(\left(Z_{k_{1},(1)}^{\left(i_{1}\right)}, Z_{k_{2}, 1}^{\left(i_{2}\right)}\right), \ldots,\left(Z_{k_{1},(N)}^{\left(i_{1}\right)}, Z_{k_{2}, N}^{\left(i_{2}\right)}\right)\right)$ elements of coupled chain $\mathbf{Z}_{k_{1}, k_{2}}^{\left(i_{1}, i_{2}\right)}$ sorted in ascending order according to the future jump 


$$
\begin{aligned}
& \text { times of } \mathbf{Z}_{k_{1}}^{\left(i_{1}\right)}, \quad \text { namely }\left(Z_{k_{1},\left(p_{1}\right)}^{\left(i_{1}\right)}, Z_{k_{2}, p_{1}}^{\left(i_{2}\right)}\right) \leq \\
& \left(Z_{k_{1},\left(p_{2}\right)}^{\left(i_{1}\right)}, Z_{k_{2}, p_{2}}^{\left(i_{2}\right)}\right) \text { if } T_{k_{1},\left(p_{1}\right)}^{\left(i_{1}\right)} \leq T_{k_{1},\left(p_{2}\right)}^{\left(i_{1}\right)}
\end{aligned}
$$

Details for the numerical assessment of the $N P V(H)$ using the AQMC method are provided in Algorithm 2.

Unlike the MC method where confidence intervals are available for the estimations, (A)QMC methods are deterministic and only an upper bound of the error is accessible through the Koksma-Hlawka theorem ${ }^{5}$, which is known to largely overestimate the error. Randomized versions are now presented, which allow to construct confidence intervals for the estimations.

\section{Randomized (Array) Quasi Monte Carlo methods}

As told in the introduction, Randomized (Array) Quasi Monte Carlo methods use randomized LDS. A randomized version of a $\operatorname{LDS}$ is a random sequence which has the two following properties ${ }^{17}$ :

1. each randomized point is uniformly distributed on $[0,1]^{d}, d \geq 1$

2. the regularity of the point is preserved (in the sense of a low discrepancy).

Thus, randomized Quasi Monte Carlo methods have advantages on both (A)QMC method (confidence intervals) and $\mathrm{MC}$ methods (better regularity than a random sample). Several randomization methods are available. We here used a random shift, which means that starting from successive points $\left(\mathbf{s}_{1}^{d}, \ldots, \mathbf{s}_{n}^{d}\right)$ of a LDS with dimension $d$, we set $\tilde{\mathbf{s}}_{i}^{d}=\left(\mathbf{s}_{i}^{d}+\mathbf{v}_{j}\right)$ $\bmod 1$ for all $1 \leq i \leq n$, where $\mathbf{v}_{j}$ is a $d$-dimensional uniform random variable ${ }^{17}$. Taking independent $\mathbf{v}_{j}$ 's provides independent trajectories for the Markov chain, allowing the construction of confidence intervals for the estimations.

As for the assessment of the $N P V(H)$, a first randomized version is obtained by substituting the deterministic LDS with dimension $m$ from Algorithm 1 (QMC method) by a randomized version. This provides the RQMC method. Two other randomized versions are considered, based on Algorithm 2 (AQMC method), which uses two LDS with respective dimensions $n+$ 1 (initialization) and 3 (transition) and simulate $N$ trajectories of the Markov chains in parallel. In both versions, the $(n+1)$-dimensional LDS is first randomized. In the first version (denoted by RAQMC), the 3-dimensional LDS is randomized one single time at the beginning, whereas it is independently randomized

\section{Algorithm 2 Array Quasi Monte Carlo to assess the NPV \\ Construction of LDS $\mathbf{S}^{n+1}$ and $\mathbf{S}^{3}$ \\ Initialization of the array of Markov chains $\mathbf{Z}_{k}^{(1)}$}

Sort elements of $\mathbf{s}_{1: N}^{n+1}$ in ascending order according to the first coordinate

Delete the first coordinate and $\mathbf{s}_{1: N}^{n}$ is obtained

$\mathbf{Z}_{0}^{(1)}=\left(Z_{0,1}^{(1)}, Z_{0,2}^{(1)}, \ldots, Z_{0, N}^{(1)}\right) \quad$ where $\quad Z_{0, i}^{(1)}=$ $\left(G\left(\left(X_{0, i, 1}, 1\right), \ldots,\left(X_{0, i, n}, 1\right)\right), 1,0,0,0,0\right) \quad$ and $X_{0, i, j}=F^{-1}\left(\mathbf{s}_{i}^{n}(j)\right), 1 \leq i \leq N, 1 \leq j \leq n$

Sort copies $\mathbf{Z}_{0}^{(1)}$ in ascending order according to the future jump times and $\mathbf{Z}_{(0)}^{(1)}$ is obtained

$\mathbf{Z}_{0}^{(1)} \leftarrow \mathbf{Z}_{(0)}^{(1)}$

$M_{\zeta-\tau}^{(1)}=\sum_{i=1}^{N} \mathbf{1}_{\left\{T_{0, i}^{(1)} \leq \zeta-\tau\right\}}$ represents the number of copies that have not reached $\zeta-\tau$

$k \leftarrow 0, m \leftarrow 1$

Simulation of CM strategy up to $\zeta-\tau$ to order spare parts in readiness for $\mathbf{P R}$

while $M_{\zeta-\tau}^{(1)}>0$ do

Sort elements of $\mathbf{s}_{m: m+M_{\zeta-\tau}^{(1)}}^{3}$ in ascending order according to the first coordinate

Delete the first coordinate: $\mathbf{s}^{2}$

$$
m: m+M_{\zeta-\tau}^{(1)}
$$

$$
\begin{aligned}
& \mathbf{Z}_{k+1}^{(1)}\left(1: M_{\zeta-\tau}^{(1)}\right) \\
& =\phi_{1}\left(\mathbf{Z}_{k}^{(1)}\left(1: M_{\zeta-\tau}^{(1)}\right), \mathbf{s}_{m: m+M_{\zeta-\tau}^{(1)}}^{2}(1)\right)
\end{aligned}
$$

$m \leftarrow m+M_{\zeta-\tau}^{(1)}$

$M_{\zeta-\tau}^{(1)} \leftarrow \sum_{i=1}^{M_{\zeta-\tau}^{(1)}} \mathbf{1}_{\left\{T_{k+1, i}^{(1)} \leq \zeta-\tau\right\}}$

Sort copies $\mathbf{Z}_{k+1}^{(1)}$ in ascending order according to the future jump times: $\mathbf{Z}_{(k+1)}^{(1)}$

$$
\begin{aligned}
& \mathbf{Z}_{k+1}^{(1)} \leftarrow \mathbf{Z}_{(k+1)}^{(1)} \\
& k \leftarrow k+1
\end{aligned}
$$

\section{end while}

Calculation of number of components which have never failed before $\zeta-\tau$

Keep lines where spare parts are not ordered, i.e. $K_{\zeta-\tau}^{(1)}=0$. These lines are placed on the vector $V_{\zeta-\tau}$

Initialization of PM strategy: $\mathrm{Z}_{0}^{(3)}$

Construction of the coupled chain

$\mathbf{Z}_{N_{\zeta-\tau}^{(1)}, 0}^{(1,3)}=\left(\mathbf{Z}_{N_{\zeta-\tau}^{(1)}}^{(1)}, \mathbf{Z}_{0}^{(3)}\right)$

Simulation of CM strategy up to horizon time $k \leftarrow N_{\zeta-\tau}^{(1)}$, thus $k$ does not have the same value for all copies

$M_{H}^{(1)}=\sum_{i=1}^{N} \mathbf{1}_{\left\{T_{0, i}^{(1)} \leq H\right\}}$ is the number of copies that have not reached $H$ 
while $M_{H}^{(1)}>0$ do

Sort elements of $\mathbf{s}_{m: m+M_{H}^{(1)}-1}^{3}$ in ascending order according to the first coordinate

Delete the first coordinate: $\mathbf{s}_{m: m+M_{H}^{(1)}-1}^{2}$

$\mathbf{Z}_{k+1}^{(1)}\left(1: M_{\zeta-\tau}^{(1)}\right)$

$=\phi_{1}\left(\mathbf{Z}_{k}^{(1)}\left(1: M_{\zeta-\tau}^{(1)}\right), \mathbf{s}_{m: m+M_{H}^{(1)}}^{2}(1)\right)$

$m \leftarrow m+M_{H}^{(1)}$

$M_{H}^{(1)} \leftarrow \sum_{i=1}^{M_{H}^{(1)}} \mathbf{1}_{\left\{T_{k+1, i}^{(1)} \leq H\right\}}$

Sort copies of coupled chain $\mathbf{Z}_{k+1,0}^{(1,3)}$ in ascending order according to the future jump times of $\mathbf{Z}_{k+1}^{(1)}$

$\mathbf{Z}_{k+1,0}^{(1,3)} \leftarrow \mathbf{Z}_{(k+1), 0}^{(1,3)}$

Give the same permutation on the vector $V_{\zeta-\tau}$ in order to keep copies where the $N P V$ is null

$k \leftarrow k+1$

end while

$$
\begin{aligned}
C_{H}^{(1)} & =C_{N_{H}^{(1)}}^{(1)} \\
& +\frac{C_{u n a}}{\alpha} \sum_{j=1}^{n}\left(e^{-\alpha X_{j, N_{H}^{(1)}}^{(1)}}-e^{-\alpha H}\right) \mathbf{1}_{\left\{X_{j, N_{H}^{(1)}}^{(1)} \leq H\right\}}
\end{aligned}
$$

Replace the lines $V_{\zeta-\tau}$ of $Z^{(3)}$ by the corresponding lines of $Z^{(1)}$

\section{Simulation of PM strategy up to time $\zeta$}

Sort copies of coupled chain $\mathbf{Z}_{N_{H}^{(1)}, 0}^{(1,3)}$ in ascending order according to the future jump times of $\mathbf{Z}_{0}^{(3)}: \mathbf{Z}_{N_{H}^{(1)},(0)}^{(1,3)}$ $\mathbf{Z}_{N_{H}^{(1)}, 0}^{(1,3)} \leftarrow \mathbf{Z}_{N_{H}^{(1)},(0)}^{(1,3)}$

Give the same permutation on the vector $V_{\zeta-\tau}$

$M_{\zeta}^{(3)} \leftarrow \sum_{i=1}^{N} \mathbf{1}_{\left\{T_{0, i}^{(3)} \leq \zeta\right\}} ; k \leftarrow 0$

while $M_{\zeta}^{(3)}>0$ do

Sort elements of $\mathbf{s}_{m: m+M_{\zeta}^{3}-1}^{3}$ in ascending order according to the first coordinate

Delete the first coordinate: $\mathbf{s}_{m: m+M_{\zeta}^{(3)}-1}^{2}$

$\mathbf{Z}_{k+1}^{(3)}\left(1: M_{\zeta}^{(3)}\right)$

$=\phi_{3}\left(\mathbf{Z}_{k}^{(3)}\left(1: M_{\zeta}^{(3)}\right), \mathbf{s}_{m: m+M_{\zeta}^{(3)}-1}^{2}(1)\right)$

$m \leftarrow m+M_{\zeta}^{(3)}$

$M_{\zeta}^{(3)} \leftarrow \sum_{i=1}^{M_{\zeta}^{(3)}} \mathbf{1}_{\left\{T_{k+1, i}^{(3)} \leq \zeta\right\}}$

Sort copies of coupled chain $\mathbf{Z}_{N_{H}^{(1)}, k+1}^{(1,3)}$ in ascending order according to the future jump times of $\mathbf{Z}_{k+1}^{(3)}$

$\mathbf{Z}_{N_{H}^{(1)}, k+1}^{(1,3)} \leftarrow \mathbf{Z}_{N_{H}^{(1)},(k+1)}^{(1,3)}$

Give the same permutation on the vector $V_{\zeta-\tau}$

$k \leftarrow k+1$

end while

\section{Preventive replacements at time $\zeta$}

The initialization of $\mathbf{Z}_{0}^{(4)}$ depends on the number of unavailable components at time $\zeta$. Denote by $N_{i, \zeta}$ the number of random variables needed for initializing the copies $Z_{0, i}^{(4)}$ then sort elements of $\mathbf{s}_{N+1: 2 N}^{n+1}$ in ascending order according to the first coordinate, delete the first coordinate and $\mathbf{s}_{N+1: 2 N}^{n}$ is obtained. The elements $S_{n}\left(N+i, 1: N_{i, \zeta}\right), 1 \leq i \leq N$, are used in order to initialize the copy $i$.

\section{Construction of coupled Markov chain}

$\mathbf{Z}_{N_{H}^{(1)}, 0}^{(1,4)}=\left(\mathbf{Z}_{N_{H}^{(1)}}^{(1)}, \mathbf{Z}_{0}^{(4)}\right)$

\section{Simulation of PM strategy up to $H$}

Sort copies of coupled chain $\mathbf{Z}_{N_{H}^{(1)}, 0}^{(1,4)}$ in ascending order according to the future jump times of $\mathbf{Z}_{0}^{(4)}: \mathbf{Z}_{N_{H}^{(1)},(0)}^{(1,4)}$ $\mathbf{Z}_{N_{H}^{(1)}, 0}^{(1,4)} \leftarrow \mathbf{Z}_{N_{H}^{(1)},(0)}^{(1,4)}$

Give the same permutation on the vector $V_{\zeta-\tau}$ $M_{H}^{(4)} \leftarrow \sum_{i=1}^{N} \mathbf{1}_{\left\{T_{0, i}^{(4)} \leq H\right\}} ; k \leftarrow 0$

while $M_{H}^{(4)}>0$ do

Sort elements of $\mathbf{s}_{m: m+M_{H}^{3}-1}^{(4)}$ in ascending order according to the first coordinate

Delete the first coordinate: $\mathbf{s}_{m: m+M_{H}^{(4)}-1}^{2}$

$$
\begin{aligned}
& \mathbf{Z}_{k+1}^{(4)}\left(1: M_{H}^{(4)}\right) \\
& =\phi_{4}\left(\mathbf{Z}_{k}^{(4)}\left(1: M_{H}^{(4)}\right), \mathbf{s}_{m: m+M_{H}^{(4)}-1}^{2}\right) \\
& m \longleftarrow m+M_{H}^{(4)} \\
& M_{H}^{(4)} \leftarrow \sum_{i=1}^{M_{H}^{(4)}} \mathbf{1}_{\left\{T_{k+1, i}^{(4)} \leq H\right\}}
\end{aligned}
$$

Sort copies of coupled chain $\mathbf{Z}_{N_{H}^{(1)}, k+1}^{(1,4)}$ in ascending order according to the future jump times of $\mathbf{Z}_{k+1}^{(4)}$

$$
\mathbf{Z}_{N_{H}^{(1)}, k+1}^{(1,4)} \leftarrow \mathbf{Z}_{N_{H}^{(1)},(k+1)}^{(1,4)}
$$

Give the same permutation on the vector $V_{\zeta-\tau}$ $k \leftarrow k+1$

\section{end while}

$$
\begin{aligned}
C_{H}^{(2)} & =C_{N_{H-\zeta}^{(4)}}^{(4)} \\
& \left.+\frac{C_{\text {una }}}{\alpha} \sum_{j=1}^{n}\left(e^{-\alpha X_{j, N_{H-\zeta}^{(4)}}^{(4)}-e^{-\alpha H}}\right) \mathbf{1}_{\substack{\left\{X_{j, N_{H-\zeta}^{(4)}}^{(4)}\\
\\
\right.}} \leq H\right\}
\end{aligned}
$$

return $N P V(H)=C_{H}^{(1)}-C_{H}^{(2)}$

at each step of the Markov chain in the second version (denoted by ARQMC).

Note that the ARQMC method is known ${ }^{16}$ to require a longer CPU time than the MC and RQMC methods 
for the same sample size but to provide estimators with a smaller variance.

\section{Numerical results on a fictitious case study}

\section{Numerical parameters}

To assess the expected NPV and the probability for the NPV to be negative at horizon time $\mathrm{H}$, the following parameters are considered:

- Operation horizon time $H=60$;

- Supply time $\tau=1$;

- Predicted time to preventive maintenance actions $\zeta=30$

- Probability distribution of components time to failure: Weibull distribution $W(48 ; 2.6)$ with c.d.f.

$$
F(x)=\left(1-e^{-\left(\frac{x}{48}\right)^{2.6}}\right) \mathbf{1}_{\{x \geq 0\}}
$$

and $F(\zeta)=1-e^{-\left(\frac{30}{48}\right)^{2.6}} \simeq 0.2552$

$$
\mathbb{E}[X] \simeq 42.6341 \text { and } \operatorname{Var}(X) \simeq 310.2542
$$

- Continuous discount rate $\alpha=0.075$;

- Corrective replacement cost $c_{r}=600 €$;

- Preventive replacement cost $c_{p}=100 €$;

- Cost of downtime $C_{i n d}=200 €$ per year;

- Purchasing price of one spare part for planned replacement $c_{s}^{p}=200 €$;

- Purchasing price of one spare part for unplanned replacement $c_{s}^{c}=600 €$.

Note that these parameters are not realistic. They have only been defined in order to develop the fictitious case study.

\section{Comparison of the different methods}

The point here is to compare the previously described methods for numerically assessing the NPV indicators. With that aim, one might use the effectiveness indicator proposed by Glynn and Whitt ${ }^{11}$, for measuring the efficiency of random simulation methods. This indicator takes into account both the variance of the estimator and the expected computing time. Later, Estécahandy ${ }^{9}$ proposed a new effectiveness indicator where the variance of the estimator is replaced by its Mean Square Error (MSE), where we recall that the MSE is the sum of the squared bias and variance of the estimator. We prefer use this second effectiveness indicator which takes into account the accuracy of the method through the bias and which can be used for deterministic methods as well (which is not the case for the original one ${ }^{11}$ ).

For a specific definition of the effectiveness indicator, let us set $\theta=\mathbb{E}[h(N P V(H))]$ to be one of the indicators of interest. Let us also introduce $\hat{\theta}^{(N)}$ to be the estimator of $\theta$ (empirical mean) based on a sample of size $N$ and let $t_{N}$ be the corresponding Central Processing Unit (CPU) time. For comparison purpose, the sample size $N$ should be the same for all envisioned numerical methods.

For the deterministic methods (QMC and AQMC), the effectiveness indicator is defined by:

$$
\varepsilon(N)=\frac{1}{\left(\hat{\theta}^{(N)}-\theta\right)^{2} \times t_{N}} .
$$

For the random methods (MC, RQMC, RAQMC and ARQMC), it is defined by:

$$
\varepsilon(N)=\frac{1}{\left(\operatorname{Var}\left(\hat{\theta}^{(N)}\right)+\left(\mathbb{E}\left(\hat{\theta}^{(N)}-\theta\right)\right)^{2}\right) \times \mathbb{E}\left(t_{N}\right)} .
$$

Note that for a deterministic method, both expressions (5) and (6) coincide. For a random method, $\varepsilon(N)$ is unknown and should be estimated. With that aim, $J$ independent simulations or randomizations of $N$ realizations of $h(N P V(H))$ are performed, which provides $J$ independent estimators $\hat{\theta}_{j}, j=1, \ldots, J$ of $\theta$ (each based on a sample of size $N$ ). These $J$ estimators are i.i.d. realizations of $\hat{\theta}^{(N)}$ (estimator of $\theta$ ). Setting $\overline{\hat{\theta}}=\frac{1}{J} \sum_{j=1}^{J} \hat{\theta}_{j}$ to be the empirical mean over $\hat{\theta}_{j}, j=$ $1, \ldots, J$, the effectiveness indicator is next estimated by:

$$
\bar{\varepsilon}=\frac{1}{\left[\frac{1}{J} \sum_{j=1}^{J}\left(\hat{\theta}_{j}-\overline{\hat{\theta}}\right)^{2}+(\overline{\hat{\theta}}-\theta)^{2}\right] \times \bar{t}}
$$

where $\bar{t}$ represents the mean of the CPU times over the $J$ independent simulations or randomizations and where the other term corresponds to the sum of the estimated variance and squared bias of the estimator, namely to the estimated MSE.

For all methods (deterministic or not), a higher effectiveness indicator reflects a better efficiency.

\section{Numerical results}

For the numerical assessment, several values are considered for the number of components $(n)$ and for the size of the initial stock $(S)$. As a first step, reference values and $95 \%$ confidence intervals (CI) are computed 
through crude MC simulations with a large sample size $N$. These reference values are denoted by $\hat{\mu}_{r e f}$ and $\hat{p}_{\text {ref }}$ for the expected $N P V(H)$ and the probability of regret $\mathbb{P}(N P V(H)<0)$, respectively.

- For $(n, S)=(5,1)$, we get with $N=10^{8}$ :

$\hat{\mu}_{\text {ref }}=16.740$ and $C I_{95 \%}\left(\hat{\mu}_{r e f}\right)=[16.724 ; 16.756]$, $\hat{p}_{\text {ref }}=0.4371$ and $C I_{95 \%}\left(\hat{p}_{\text {ref }}\right)=[0.437 ; 0.4372]$.

- For $(n, S)=(10,2)$, we get with $N=2 \times 10^{8}$ :

$\hat{\mu}_{\text {ref }}=33.573$ and $C I_{95 \%}\left(\hat{\mu}_{\text {ref }}\right)=[33.558 ; 33.584]$, $\hat{p}_{\text {ref }}=0.396$ and $C I_{95 \%}\left(\hat{p}_{\text {ref }}\right)=[0.3959 ; 0.3961]$.

- For $(n, S)=(20,4)$, we get with $N=5 \times 10^{8}$ :

$\hat{\mu}_{\text {ref }}=70.998$ and $C I_{95 \%}\left(\hat{\mu}_{\text {ref }}\right)=[70.983 ; 71.012]$, $\hat{p}_{\text {ref }}=0.3394$ and $C I_{95 \%}\left(\hat{p}_{\text {ref }}\right)=[0.33936 ; 0.33944]$.

The positive values of $\hat{\mu}_{\text {ref }}$ illustrate the fact that, in a mean behavior, the PM strategy enables to reduce unplanned unavailabilities. However, in view of the probability of regret, at least one history over three leads to a higher cost under the PM strategy than under the purely corrective one. The decision to use or not the PM strategy hence depends on the risk aversion of the decision maker.

We now come back to the point of the present paper, which is the comparison of the numerical results obtained by the different methods described previously. with that aim, we set:

- $\varepsilon_{\text {exp }}$ and $\bar{\varepsilon}_{\text {exp }}$ : the effectiveness indicator on the expected $N P V(H)$ for the deterministic and random methods, respectively;

- $\varepsilon_{p}$ and $\bar{\varepsilon}_{p}$ : the effectiveness indicator on the probability for the $N P V$ to be negative at time $H$ for the deterministic and random methods, respectively.

Deterministic methods are first compared in Table 1 , which provides the effectiveness indicators of both QMC and AQMC methods. As can be seen in Table 1, the effectiveness of the QMC and AQMC methods highly depends on the number of components $(n)$ and on the size of initial stock $(S)$, and also on the number $N$ of elements of LDS used for the computations. The same remark is still valid for the respective efficiency of QMC and AQMC: sometimes, QMC is better than AQMC,
Table 1. Comparison of deterministic methods

\begin{tabular}{|c|c|c|c|c|}
\hline & & Methods & $\varepsilon_{e x p}$ & $\varepsilon_{p}\left(\times 10^{4}\right)$ \\
\hline \multirow{4}{*}{ 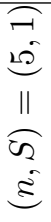 } & $N=2^{15}$ & $\mathrm{QMC}$ & 22.719 & 20160 \\
\hline & $(32768)$ & AQMC & 157.423 & 237.199 \\
\hline & $N=2^{16}$ & $\mathrm{QMC}$ & 415.246 & 90.983 \\
\hline & $(65536)$ & AQMC & 394.231 & 6.052 \\
\hline \multirow{4}{*}{ 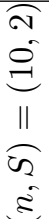 } & $N=2^{18}$ & $\mathrm{QMC}$ & 159.462 & 159.458 \\
\hline & $(262144)$ & AQMC & 188.849 & 129.356 \\
\hline & $N=2^{19}$ & $\mathrm{QMC}$ & 186.392 & 44.593 \\
\hline & $(524288)$ & AQMC & 40.359 & 495.617 \\
\hline \multirow{4}{*}{ 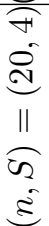 } & $N=2^{19}$ & QMC & 7.944 & 2.173 \\
\hline & $(524288)$ & AQMC & 1.957 & 452.940 \\
\hline & $N=2^{20}$ & QMC & 3.053 & 1.380 \\
\hline & $(1048576)$ & AQMC & 34.258 & 1.292 \\
\hline
\end{tabular}

Table 2. Comparison of random methods

\begin{tabular}{|c|c|c|c|c|}
\hline & & Methods & $\bar{\varepsilon}_{\text {exp }}$ & $\bar{\varepsilon}_{p}\left(\times 10^{4}\right)$ \\
\hline \multirow{8}{*}{ 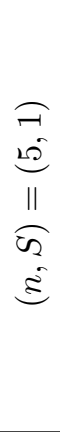 } & \multirow{4}{*}{$\begin{array}{l}N=2^{15} \\
(32768)\end{array}$} & $\mathrm{MC}$ & 1.867 & 5.641 \\
\hline & & RQMC & 36.972 & 18.815 \\
\hline & & RAQMC & 71.478 & 14.119 \\
\hline & & ARQMC & 53.431 & 14.458 \\
\hline & \multirow{4}{*}{$\begin{array}{l}N=2^{16} \\
(65536)\end{array}$} & $\mathrm{MC}$ & 2.018 & 5.111 \\
\hline & & RQMC & 27.254 & 13.610 \\
\hline & & RAQMC & 46.099 & 13.415 \\
\hline & & ARQMC & 68.175 & 15.476 \\
\hline \multirow{8}{*}{ 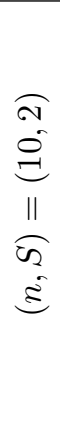 } & \multirow{4}{*}{$\begin{array}{c}N=2^{18} \\
(262144)\end{array}$} & $\mathrm{MC}$ & 0.5425 & 2.883 \\
\hline & & RQMC & 10.595 & 7.181 \\
\hline & & RAQMC & 14.078 & 6.735 \\
\hline & & ARQMC & 14.144 & 5.115 \\
\hline & \multirow{4}{*}{$\begin{array}{c}N=2^{19} \\
(524288)\end{array}$} & $\mathrm{MC}$ & 0.572 & 3.764 \\
\hline & & RQMC & 8.848 & 1.057 \\
\hline & & RAQMC & 11.816 & 5.280 \\
\hline & & ARQMC & 10.967 & 7.785 \\
\hline \multirow{8}{*}{ 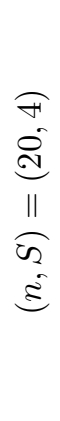 } & \multirow{4}{*}{$\begin{array}{c}N=2^{18} \\
(262144)\end{array}$} & $\mathrm{MC}$ & 0.082 & 1.140 \\
\hline & & RQMC & 1.226 & 2.919 \\
\hline & & RAQMC & 2.715 & 2.762 \\
\hline & & ARQMC & 2.762 & 2.667 \\
\hline & \multirow{4}{*}{$\begin{array}{c}N=2^{19} \\
(524288)\end{array}$} & $\mathrm{MC}$ & 0.110 & 1.916 \\
\hline & & RQMC & 1.632 & 2.940 \\
\hline & & RAQMC & 2.453 & 2.556 \\
\hline & & ARQMC & 2.314 & 1.841 \\
\hline
\end{tabular}

sometimes, it is the contrary. It is hence difficult to identify the most efficient deterministic method. 
For a better understanding of the respective efficiency of QMC and AQMC methods, we now compare their randomized versions. With that aim, $J=128$ independent sets of $N$ trajectories have been simulated for each method. The results are provided in Table 2. For the expected NPV, Table 2 shows that the randomized versions of AQMC method (RAQMC and ARQMC methods) have always an effectiveness indicator higher than that of randomized version of QMC method (RQMC method). For example, for $(n, S)=(10,2)$ and $N=2^{18}=262144$, the effectiveness indicator on the expected NPV is equal to 14.078 for the RAQMC method, 14.144 for ARQMC method while it equals to 10.595 for the RQMC method, exhibiting an efficiency gain of more than $30 \%$. This efficiency gain is higher than $121 \%$ for $(n, S)=(20,4)$ and $N=2^{18}=262144$. These results reflect that RAQMC and ARQMC methods are more efficient than RQMC method for the estimation of the expected NPV. The effectiveness of the QMC method for estimating the probability of regret is confirmed with the randomization. In fact, RQMC method is often the most effective for this indicator. However, we can emphasize that all randomized quasi Monte Carlo methods are more effective than the MC method whatever the number of components $(n)$, the initial stock $(S)$ and the number of realizations $(N)$ with an efficiency factor for the expected NPV up to 38 with $(n, S)=(5,1)$ and $N=2^{15}=32768$ for example.

\section{Conclusion}

Two quasi Monte Carlo methods have been proposed for the numerical assessment of complex PDMPs. In the first quasi Monte Carlo method (QMC), the dimension of the LDS is linked to the number of steps of the PDMPs and consequently, to the number of components as well. This is restricted to fleets with a few components because in the opposite case, the quality of the LDS decreases and also, memory problems may happen. In AQMC method, the dimension of the LDS is equal to the sum of the dimension of the Markov chain and of the number of random variables required for one single event. That significantly reduces the dimension of the LDS.

Based on the results of the present paper and on other numerical experiments not provided here, it seems that QMC, AQMC methods and their randomized version are promising alternatives to the most standard MC method for the numerical assessment of PDMPs. To be more specific, in our context, we have observed that the QMC method is often the most effective for a fleet of less than 10 components. The QMC method is then recommended for this size of fleet. Moreover, the adjustment of the MC method to obtain the QMC method is simple. The AQMC method should be preferred for a larger fleet (more than 10 components). In fact, the dimension of the LDS stays low when the number of components increases. The effectiveness of these methods should be greatly appreciated within a preventive maintenance optimization algorithm. It is important to highlight that the developments that have been presented here could be adapted for the assessment of other maintenance strategies or for computing other reliability indicators. We can then imagine that the classical black box MC simulator of industrial reliability softwares could be replaced by a black box (AR)QMC simulator.

\section{References}

1. Berk J and DeMarzo P. Corporate Finance, Pearson Education, 2013.

2. Black J and Hashimzade $\mathrm{N}$ and Myles G. A dictionary of economics , 3rd ed. Oxford : Oxford University Press, 2009.

3. Brandejsky A and De Saporta B and Dufour F. Numerical method for expectations of Piecewise Determistic Markov Processes. Communications in Applied Mathematics and Computational Science 2012; 7(1): 63-104.

4. Barringer H.P and Monroe T.R. How to justify machinery improvements using reliability engineering principles. Proceedings of the sixteenth international pump users symposium; Turbomachinery Laboratory, Texas A\&M University, College Station, Texas; 1999; 171-194.

5. Caflisch R. E. Monte-Carlo and quasi Monte-Carlo methods. Acta Numerica 1998; 7: 1-49.

6. Davis M. H. A. Piecewise Deterministic Markov Processes: A general class of non diffusion stochastic models. Journal of the Royal Statistical Society. Series $B$ (Methodological) 1984; 46(3): 353-388.

7. Ding SH and Kamaruddin S. Maintenance policy optimization - literature review and directions. The International Journal of Advanced Manufacturing Technology 2015; 76(5):1263-1283.

8. El Haddad R and Lécot C and L'Ecuyer P and Nassif N. Quasi Monte-Carlo methods for Markov chains with 
continuous multidimensional state space. Mathematics and Computers in Simulation 2010, 81: 560-567.

9. Estécahandy M. Méthodes accélérées de Monte-Carlo pour la simulation d'événements rares. Applications aux Réseaux de Petri. PhD Thesis, University of Pau and Pays de l'Adour, France, 2016.

10. Eymard R and Mercier S and Prignet A. An implicit finite volume scheme for a scalar hyperbolic problem with measure data related to Piecewise Deterministic Markov Processes Journal of Computational and Applied Mathematics 2008; 222(2):293-323.

11. Glynn P. W and Whitt W. The Asymptotic Efficiency of Simulation Estimators. Operations research 1992; 40(3):505-520.

12. Krykova I. Evaluating of Path-Dependent Securities with Low Discrepancy Methods. Master's thesis, Worcester polytechnic institute, 2003.

13. Lair W et al. Piecewise Deterministic Markov Processes and maintenance modeling: application to maintenance of a train air-conditioning system. Proc IMechE, Part O: Journal of Risk and Reliability 2011; 225(2):199-209.

14. Lécot C and Tuffin B. Quasi Monte-Carlo methods for estimating transient measures of discrete time Markov chains. In: Monte Carlo and Quasi-Monte Carlo Methods in Scientific Computing, Singapore, 25-28 November 2002, pp.329-343. Springer Berlin Heidelberg.

15. Lécot $\mathrm{C}$ and L'Ecuyer $\mathrm{P}$ and L'Archevêque-Gaudet $\mathrm{A}$. On array-RQMC for Markov chains: mapping alternatives and convergence rates. In: Monte Carlo and QuasiMonte Carlo Methods 2008, Montréal,Canada, 2009, pp.485-500. Springer.

16. L'Ecuyer P and Lécot C and Tuffin B. A Randomized Quasi-Monte Carlo simulation method for Markov Chains. Operations research 2008; 56(4):958-975.

17. Lemieux C. Monte Carlo and Quasi Monte-Carlo Sampling, Mathematics and Statistics, Springer-Verlag New York, 2009

18. Marais K.B and Saleh J.H. Beyond its cost, the value of maintenance: An analytical framework for capturing its net present value Reliability Engineering \& System Safety 2009; 94(2): 644-657.

19. Niederreiter H. Random number generation and quasi Monte-Carlo methods, Society for Industrial and Applied Mathematics, Philadelphia, PA, USA, 1992.

20. Pagès $\mathrm{G}$ and Pham $\mathrm{H}$ and Printems J. Optimal quantization methods and applications to numerical problems in finance. In: Handbook on Numerical Methods in Finance, Birkhäuser, Boston, MA, 2004.
21. Saleh J.H and Marais K.B. Reliability: How much is it worth? Beyond its estimation or prediction, the (net) present value of reliability. Reliability Engineering \&6 System Safety 2006; 91(6): 665-673.

22. Zhang $\mathrm{H}$ et al. Piecewise Deterministic Markov Processes and dynamic reliability. Proc IMechE, Part O: Journal of Risk and Reliability 2009; 222(4):545-551. 


\section{Appendix A: Transition function of Markov chain under CM strategy on $[0, H]$}

We set $F$ to be the common c.d.f of the components lifetimes. Setting $U \sim \mathcal{U}([0,1])$, we recall that $F^{-1}(U)$ stands for a random variable with c.d.f. $F$ and hence stands for a lifetime. We also set $(x, i)=$ $\left(\left(x_{1}, i_{1}\right), \ldots,\left(x_{n}, i_{n}\right)\right)$ and use $(x, i)$ as a shortcut. Finally, $G(x, i)$ stands for vector $\left(\left(x_{1}, i_{1}\right), \ldots,\left(x_{n}, i_{n}\right)\right)$ sorted by ascending order according to the $x_{i}$ 's. These notations are used in all the following appendixes.

We here present the initialization and transition function $\phi_{1}$ introduced in Equation (1) for the modelling of the CM strategy. As for the initialization, we have:

- $\left(X_{0}^{(1)}, I_{0}^{(1)}\right)=G\left(\left(F^{-1}\left(U_{1}\right), 1\right), \ldots,\left(F^{-1}\left(U_{n}\right), 1\right)\right)$ where $U_{1}, \ldots, U_{n}$ are independent uniform random variables on $[0,1]$,

- $S_{0}^{(1)}=S$ : the stock contains $S$ spare parts at initial time,

- $D_{0}^{(1)}=0$ : no order is in progress at initial time,

- $L_{0}^{(1)}=0, C_{0}^{(1)}=0, T_{0}^{(1)}=0$.

Based on the fact that no spare part can be ordered after $H-\tau$, the transitions of $\left(Z_{k}^{(1)}\right)_{k>0}$ are different before and after $H-\tau$ and we consequently distinguish the two cases.

\section{Case 1: the future jump of the Markov chain is} before $H-\tau$.

In this case, a new spare part is ordered at each failure time. As for the transitions, there are two cases, according to whether the stock is empty or not.

Firstly, assume that the stock contains $s>0$ spare parts. This induces that all components are working and there may be orders in progress. We distinguish two possibilities: the stock contains $S$ spare parts or the stock contains $s<S$ spare parts. The transition function is then defined by:

$$
\begin{aligned}
& \phi_{1}(((x, i), s, d, 0, c, t), u) \\
& =\varphi_{1}^{a}(((x, i), s, d, 0, c, t), u) \mathbf{1}_{\{s=S\}} \\
& +\varphi_{1}^{b}(((x, i), s, d, 0, c, t), u) \mathbf{1}_{\{0<s<S\}}
\end{aligned}
$$

- Possibility 1: the stock contains $S$ spare parts. In this case, no order is in progress. Thus, the only event that may occur is a failure. As one spare part is available, the failed component is replaced and its new time for future failure is randomly drawn.
The failure times are next sorted afresh according to $G$. Also, a new spare part is ordered, which will arrive after the supply time $\tau$. Finally, the cost of the $\mathrm{CR}$ and of the spare part are added to the cumulative cost. Then:

$$
\begin{aligned}
& \varphi_{1}^{a}(((x, i), S, 0,0, c, t), u) \\
& =\left(G(\bar{x}, \bar{i}), S-1, x_{1}+\tau, 0, c+C_{c} \times e^{-\alpha x_{1}}, x_{1}\right)
\end{aligned}
$$

where

$$
\begin{aligned}
& (\bar{x}, \bar{i})=\left(\left(x_{1}+F^{-1}(u), 0\right),\left(x_{2}, i_{2}\right), \ldots,\left(x_{n}, i_{n}\right)\right) \\
& C_{c}=c_{c}+c_{s}^{c} .
\end{aligned}
$$

- Possibility 2: the stock contains $0<s<S$ spare parts. This induces that at least one order is in progress. If a spare part arrive before a failure of a component, as all components are working, the stock is then supplied. If a failure occurs before a delivery of a spare part, then the failed component is replaced by a new one and its future failure time is randomly drawn. The failure times are next sorted afresh according $G$. A new spare part is ordered and the cumulative cost is updated. We obtain:

$$
\begin{aligned}
& \varphi_{1}^{b}\left(\left((x, i), s,\left(d_{1}, \ldots, d_{m}\right), 0, c, t\right), u\right) \\
= & \left((x, i), s+1,\left(d_{2}, \ldots, d_{m}\right), 0, c, d_{1}\right) \mathbf{1}_{\left\{x_{1}>d_{1}\right\}} \\
+ & \left(G(\bar{x}, \bar{i}), s-1, \bar{d}, 0, c+C_{c} \times e^{-\alpha x_{1}}, x_{1}\right) \mathbf{1}_{\left\{x_{1} \leq d_{1}\right\}}
\end{aligned}
$$

where

$$
\begin{aligned}
& (\bar{x}, \bar{i})=\left(\left(x_{1}+F^{-1}(u), 0\right),\left(x_{2}, i_{2}\right), \ldots,\left(x_{n}, i_{n}\right)\right), \\
& C_{c}=c_{c}+c_{s}^{c}, \\
& \bar{d}=\left(d, x_{1}+\tau\right) .
\end{aligned}
$$

Secondly, assume that the stock is empty. Then, at least one order is in progress in order to provide the stock. There are two possible configurations: either some components are working or all components are down. Denote by $-s$ the number of failed components, $x=\left(x_{1}, \ldots, x_{n}\right)$ the times for last or future failure of components (depending on whether the components are down or up, respectively), sorted in ascending order and $d=\left(d_{1}, \ldots, d_{m}\right)$ the predicted times for spare parts arrivals. Note that the next failure occurs at the lowest time greater than the current time i.e. at time $x_{1-s}$. If there are some up components $(-s<n)$, two events may happen: either a failure $\left(x_{1-s} \leq d_{1}\right)$ or the delivery of a spare part $\left(x_{1-s}>d_{1}\right)$. If all components are down 
$(-s=n)$, the only possible event is the delivery of a spare part. Based on this analysis, there are three possible scenarios:

$$
\begin{aligned}
& \phi_{1}(((x, i), s, d, 0, c, t), u) \mathbf{1}_{\{s \leq 0\}} \\
& =\phi_{1}^{a}(((x, i), s, d, 0, c, t), u) \mathbf{1}_{\left\{x_{1-s} \leq d_{1},-n<s \leq 0\right\}} \\
& +\phi_{1}^{b}(((x, i), s, d, 0, c, t), u) \mathbf{1}_{\left\{x_{1-s}>d_{1},-n<s \leq 0\right\}} \\
& +\phi_{1}^{c}(((x, i), s, d, 0, c, t), u) \mathbf{1}_{\{s=-n\}}
\end{aligned}
$$

which we now look at specifically.

- Scenario a: there are up components and a failure occurs before the delivery of a spare part $\left(x_{1-s} \leq\right.$ $\left.d_{1},-n<s \leq 0\right)$. As the stock is empty, the failed component becomes unavailable and an order is carried out for its replacement. Thus, the number of unavailable components is increased by 1 and the purchasing price of one spare part is added to the cumulative cost:

$$
\begin{aligned}
& \phi_{1}^{a}(((x, i), s, d, 0, c, t), u) \\
& =\left((\bar{x}, \bar{i}), s-1, \bar{d}, 0, c+c_{A} \times e^{-\alpha x_{1-s}}, x_{1-s}\right)
\end{aligned}
$$

with

$$
\begin{aligned}
& (\bar{x}, \bar{i})=\left(x_{1}, i_{1}\right), \ldots,\left(x_{1-s}, 0\right), \ldots,\left(x_{n}, i_{n}\right), \\
& \bar{d}=\left(d, x_{1-s}+\tau\right) .
\end{aligned}
$$

- Scenario b: there are up components and the delivery of a spare part occurs before a failure $\left(x_{1-s}>d_{1},-n<s \leq 0\right)$. If all components are working, then the stock is supplied and it contains now one spare part. Consequently, there is no more order in progress. In the opposite case (at least one down component), a CR is performed on the down component which has the oldest failure time and its new time for future failure is drawn. The number of unavailable components is decreased by 1. Finally, the failure times are sorted afresh and the costs of $\mathrm{CR}$ and downtime are added to the cumulated cost:

$$
\begin{aligned}
& \phi_{1}^{b}(((x, i), s, d, 0, c, t), u) \\
& =\left((x, i), 1,0,0, c, d_{1}\right) \mathbf{1}_{\{s=0\}} \\
& +\left(G(\tilde{x}, \tilde{i}), s+1,\left(d_{2}, \ldots, d_{m}\right), 0, c+\bar{C}, d_{1}\right) \mathbf{1}_{\{s<0\}}
\end{aligned}
$$

- Scenario $c$ : all components are down $(s=-n)$. The next event is the delivery of a spare part. The $\mathrm{CR}$ of the component which has the oldest failure time is performed:

$$
\begin{aligned}
& \phi_{1}^{c}(((x, i), s, d, 0, c, t), u) \\
& =\left(G(\tilde{x}, \tilde{i}), s+1,\left(d_{2}, \ldots, d_{m}\right), 0, c+\bar{C}, d_{1}\right)
\end{aligned}
$$

with

$$
\begin{aligned}
& (\tilde{x}, \tilde{i})=\left(\left(d_{1}+F^{-1}(u), 0\right),\left(x_{2}, i_{2}\right), \ldots,\left(x_{n}, i_{n}\right)\right), \\
& C=c_{c} \times e^{-\alpha d_{1}}+C_{\text {una }} \int_{x_{1}}^{d_{1}} e^{-\alpha t} d t \\
& =c_{c} \times e^{-\alpha d_{1}}+\left(C_{\text {una }} / \alpha\right) \times\left(e^{-\alpha x_{1}}-e^{-\alpha d_{1}}\right) .
\end{aligned}
$$

\section{Case 2: the future jump of the Markov chain is after $H-\tau$.}

The only difference is that no spare part is ordered at failure times. However, the previously ordered spare parts may still arrive. Once empty, the stock remains empty up to horizon $H$.

\section{Appendix B: Transition function of Markov chain under PM strategy on $(\zeta-\tau, \zeta)$}

We only consider the case where at least one spare parts is ordered at time $\zeta-\tau$ (because both $\mathrm{CM}$ and $\mathrm{PM}$ strategies coincides in the other case). We recall that the Markov chain $Z^{(3)}$ models the evolution of the fleet of components under the PM strategy on $[\zeta-\tau, \zeta)$ and that $K_{\zeta-\tau}^{(1)}=\sum_{j=1}^{n} I_{j, N_{\zeta-\tau}^{(1)}}^{(1)}$ spare parts are ordered at time $\zeta-\tau$, which will arrive at time $\zeta$. We begin with the initialization of $Z^{(3)}$ which only depends on the state of $Z^{(1)}$ at time $\zeta-\tau$ (or at time $T_{N_{\zeta-\tau}^{(1)}}^{(1)}$, equivalently):

$$
\begin{aligned}
& Z_{0}^{(3)}=\left(\left(X_{N_{\zeta-\tau}^{(1)}}^{(1)}, I_{N_{\zeta-\tau}^{(1)}}^{(1)}\right), S_{N_{\zeta-\tau}^{(1)}}^{(1)},\left(D_{N_{\zeta-\tau}^{(1)}}^{(1)}, \zeta\right)\right. \\
& \left.L_{N_{\zeta-\tau}^{(1)}}^{(1)}, C_{N_{\zeta-\tau}^{(1)}}^{(1)}, \zeta-\tau\right)
\end{aligned}
$$

where $\zeta$ is added to the dates of spare parts arrivals.

Let us recall that, in the PM strategy, a new spare part is ordered at failure of one component on $(\zeta-\tau, \zeta)$ only if the failed component has already been replaced (because in the opposite case, a spare part has already been ordered at time $\zeta-\tau$ in view of its $\mathrm{PR}$ at atime $\zeta)$. As a consequence, the transitions of $Z^{(3)}$ differ from that of $Z^{(1)}$ only at failure of a component which has not yet suffered from any failure $\left(I_{j, t}^{(1)}=1\right)$ and for which no spare part is ordered when it fails. We consequently only consider this case here and refer to the transitions of $Z^{(1)}$ for all other transitions. Two different cases have to be envisioned, according to whether the stock is empty or not at the failure time. When the stock is not empty 
$(s>0)$, a CR takes place, which provides:

$$
\begin{aligned}
& \phi_{3}\left(\left(\left(\left(x_{1}, 1\right),\left(x_{2}, i_{2}\right), \ldots,\left(x_{n}, i_{n}\right)\right), s, \zeta, 0, c, t\right), u\right) \\
& =\left(G(\bar{x}, \bar{i}), s-1, \zeta, 0, c+c_{c} \times e^{-\alpha x_{1}}, x_{1}\right)
\end{aligned}
$$

with

$$
(\bar{x}, \bar{i})=\left(\left(x_{1}+F^{-1}(u), 0\right),\left(x_{2}, i_{2}\right), \ldots,\left(x_{n}, i_{n}\right)\right) .
$$

When it is empty $(s \leq 0)$, no CR takes place and the PR of the failed component is cancelled. Noting that the index of the failed component is $1-s$ and we get:

$$
\begin{aligned}
& \phi_{3}\left(\left(\left(\left(x_{1}, i_{1}\right), \ldots,\left(x_{1-s}, 1\right) \ldots,\left(x_{n}, i_{n}\right)\right), s, d, 0, c, t\right), u\right) \\
& =\left((\tilde{x}, \tilde{i}), s-1, d, 0, c, x_{1-s}\right)
\end{aligned}
$$

with

$$
(\tilde{x}, \tilde{i})=\left(\left(x_{1}, i_{1}\right), \ldots,\left(x_{1-s}, 0\right), \ldots,\left(x_{n}, i_{n}\right)\right) .
$$

\section{Appendix C: Transition function of Markov chain under PM strategy on $[\zeta, H]$}

The spare parts which have been ordered at time $\zeta-\tau$ arrive at time $\zeta$. They are used first for potential pending $\mathrm{CR}$ and next for PR. The Markov chain $Z^{(4)}$ models the fleet of components on $[\zeta, H)$ under the PM strategy and its initialization depends on the number of unavailable components and of the components awaiting a PR at time $\zeta$. Consequently, the initialization of $Z^{(4)}$ is a deterministic function of all variables at time $\zeta^{-}$, namely of $Z_{\zeta}^{(3)}$ or $Z_{N_{\tau}^{(3)}}^{(3)}$. In the PM strategy, an uniform random variable is used to draw a new lifetime at each failure time if a spare part is available. However, recall that in case where we have postponed PR, an additional uniform random variable may be necessary for the eventual choice of component to be preventively replaced. The transition function of $\left(Z^{(4)}\right.$ then depends on a random uniform variable of dimension one or two and is defined by:

$$
\left\{\begin{array}{l}
Z_{0}^{(4)}=\left(G\left(X_{0}^{(4)}, I_{0}^{(4)}\right), S_{0}^{(4)}, D_{0}^{(4)}, L_{0}^{(4)}, C_{0}^{(4)}, \zeta\right) \\
Z_{k+1}^{(4)}=\phi_{4}\left(Z_{k}^{(4)}, U_{k+1}\right) ; k \geq 0
\end{array}\right.
$$

where $U_{k+1}=\left(U_{k+1}(1), U_{k+1}(2)\right) \sim \mathcal{U}\left([0,1]^{2}\right)$.

Let $K_{\zeta}^{(3)}=\sum_{j=1}^{n} I_{j, \zeta}^{(3)}$ be the number of components which have never been replaced. The different cases of initialization of Markov chain $Z^{(4)}$ are given in the following subsections.

\section{All components are working at time $\zeta$ $\left(S_{N_{\tau}^{(3)}}^{(3)} \geq 0\right)$}

In this case, all components awaiting a PR will be replaced. Their new time for future failure is randomly drawn and the failure times are sorted afresh according $G$. If there is unused spare parts, they are added in the stock. The cost of PR and the purchase cost of spare part for PR are added on the cost just before $\zeta$. We the obtain:

- $X_{0}^{(4)}=\left(X_{1,0}^{(4)}, \ldots, X_{n, 0}^{(4)}\right)$ where for $j \in\{1, \ldots, n\}$ and $u \sim \mathcal{U}([0,1])$,

$$
X_{j, 0}^{(4)}=\left\{\begin{array}{l}
\zeta+F^{-1}(u) \quad \text { if } I_{j, N_{\tau}^{(3)}}^{(3)}=1 \\
X_{j, N_{\tau}^{(3)}}^{(3)} \quad \text { otherwise }
\end{array}\right.
$$

the new failure times of all components which have never been replaced $\left(I_{j, N_{\tau}^{(3)}}^{(3)}=1\right)$ are drawn. $N_{\tau}^{(3)}$ stands for the number of jumps of $Z^{(3)}$ on $(\zeta-\tau, \zeta)$

- $I_{0}^{(4)}=(0, \ldots, 0)$ : all components which have not suffered from any failure are preventively replaced at time $\zeta$. Then, all components have been replaced $(\mathrm{CR}$ or $\mathrm{PR})$ at time $\zeta$;

- $S_{0}^{(4)}=S_{N_{\tau}^{(3)}}^{(3)}+K_{\zeta-\tau}-K_{\zeta}$ : the unused spare parts are added in the stock;

- $D_{0}^{(4)}=\left(D_{2, N_{\tau}^{(3)}}^{(3)}, \ldots, D_{m, N_{\tau}^{(3)}}^{(3)}\right)$ : the spare parts arrive at $\zeta$ and $D_{1, N_{\tau}^{(3)}}^{(3)}=\zeta$ is removed in $D_{N_{\tau}^{(3)}}^{(3)}$;

- $L_{0}^{(4)}=0$ : no deferred PR;

- $C_{0}^{(4)}=C_{N_{\tau}^{(3)}}^{(3)}+\left(K_{\zeta-\tau}^{(1)} \times c_{s}^{p}+K_{\zeta}^{(3)} \times c_{p}\right) \times e^{-\alpha \zeta}$ : the cost of PR and of spare parts are added on the cost just before $\zeta$.

As all components have been replaced at time $\zeta$, the transition function under PM strategy after time $\zeta$ evolves in the same way as CM strategy. Then, the transition function of Markov chain $Z^{(4)}$ is defined by:

$Z_{k+1}^{(4)}=\phi_{4}\left(Z_{k}^{(4)}, U_{k+1}\right)=\phi_{1}\left(Z_{k}^{(4)}, U_{k+1}(1)\right) ; k \geq 0$

\section{There are some unavailable components at $\zeta$ $\left(S_{N_{\tau}^{(3)}}^{(3)}<0\right)$}

Here, the components awaiting a CR are first repaired. There are two different cases whether the number of spare parts arrivals $\left(K_{\zeta-\tau}^{(1)}\right)$ is lower or equal to the number of components awaiting a corrective or preventive replacement $\left(K_{\zeta}^{(3)}-S_{N_{\tau}^{(3)}}^{(3)}\right)$. Note that this case where the components are unavailable at $\zeta$, the number of spare parts arrivals at time $\zeta$ cannot be upper 
than the number of awaiting replacements. In fact, on $(\zeta-\tau, \zeta)$ a spare part is ordered only at the failure of a component which has already suffered a failure before and it will be delivered after $\zeta$. Let $\chi_{\zeta}$ be the subset of subscripts of components which have never been replaced up to $\zeta \cdot \chi_{\zeta}$ is defined by:

$$
\chi_{\zeta}=\left\{j \in\{1, \ldots, n\}: I_{j, N_{\tau}^{(3)}}^{(3)}=1\right\} .
$$

We can note that $\operatorname{card}\left(\chi_{\zeta}\right)=K_{\zeta}^{(3)}$

Case 1: the number of spare parts arrivals is equal to the number of components awaiting a replacement $\left(K_{\zeta}^{(3)}-S_{\zeta^{-}}^{(3)}=K_{\zeta-\tau}^{(1)}\right)$. In this case, all components awaiting a replacement will be replaced. We obtain:

- $X_{0}^{(4)}=\left(X_{1,0}^{(4)}, \ldots, X_{n, 0}^{(4)}\right)$ where for $j \in\{1, \ldots, n\}$ and $u \sim \mathcal{U}([0,1])$,

$$
X_{j, 0}^{(4)}=\left\{\begin{array}{l}
\zeta+F^{-1}(u) \text { if } 1 \leq j \leq-S_{j, N_{\tau}^{(3)}}^{(3)} \\
\zeta+F^{-1}(u) \text { if } j \in \chi_{\zeta} \\
X_{j, N_{\tau}^{(3)}}^{(3)} \quad \text { otherwise }
\end{array}\right.
$$

all $\mathrm{CR}$ and $\mathrm{PR}$ are performed, their predicted times for future failure are drawn and the other components are not changed;

- $I_{0}^{(4)}=(0, \ldots, 0)$ : all components have been replaced $(\mathrm{CR}$ or $\mathrm{PR})$ at time $\zeta$;

- $S_{0}^{(4)}=0$ : all components are working after replacements and all spare parts arrivals have been used;

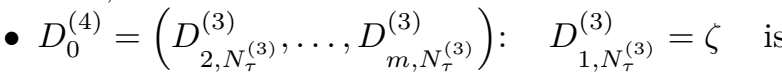
removed;

- $L_{0}^{(4)}=0$ : no deferred PR;

- The cost of CR, of PR and of downtime of components are added to the cumulated cost:

$$
\begin{aligned}
C_{0}^{(4)} & =C_{N_{\tau}^{(3)}}^{(3)}+\left(K_{\zeta-\tau}^{(1)} \times c_{s}^{p}+K_{\zeta}^{(3)} \times c_{p}\right) \times e^{-\alpha \zeta} \\
& -S_{N_{\tau}^{(3)}}^{(3)} \times c_{c} \times e^{-\alpha \zeta}+C_{\text {una }}^{\zeta}
\end{aligned}
$$

with

$$
C_{u n a}^{\zeta}=\left(C_{u n a} / \alpha\right) \times \sum_{j=1}^{-S_{\tau}^{(3)}}\left(e^{\alpha X_{j, N_{\tau}^{(3)}}^{(3)}}-e^{-\alpha \zeta}\right) .
$$

As previous case, no $\mathrm{PR}$ is deferred. Then, the transition function of Markov chain $Z^{(4)}$ is given by:

$$
Z_{k+1}^{(4)}=\phi_{4}\left(Z_{k}^{(4)}, U_{k+1}\right)=\phi_{1}\left(Z_{k}^{(4)}, U_{k+1}(1)\right) ; k \geq 0 .
$$

Case 2: the number of spare parts arrivals is lower than the number of components awaiting a replacement $\left(K_{\zeta}^{(3)}-S_{N_{\tau}^{(3)}}^{(3)}>K_{\zeta-\tau}^{(1)}\right)$. Here, the PR will be deferred. $\mathrm{CR}$ are first carried out. If there are remaining spare parts, the $\mathrm{PR}$ are performed as long as the spare parts are available. Let $N_{c}^{\zeta}=\min \left(-S_{N_{\tau}^{(3)}}^{(3)}, K_{\zeta-\tau}^{(1)}\right)$ be the number of components which will be correctively replaced at $\zeta$ and $\bar{\chi}_{\zeta}$ be the subscripts of components which will be preventively replaced at time $\zeta$ if there are remaining spare parts $N_{p}^{\zeta}=\left(K_{\zeta-\tau}^{(1)}+S_{N_{\tau}^{(3)}}^{(3)}>0\right)$. We have:

- $X_{0}^{(4)}=\left(X_{1,0}^{(4)}, \ldots, X_{n, 0}^{(4)}\right)$ where for $j \in\{1, \ldots, n\}$ and $u \sim \mathcal{U}([0,1])$,

$$
X_{j, 0}^{(4)}=\left\{\begin{array}{l}
\zeta+F^{-1}(u) \text { if } 1 \leq j \leq N_{c}^{\zeta} \\
\zeta+F^{-1}(u) \text { if } N_{p}^{\zeta}>0 \text { and } j \in \bar{\chi}_{\zeta} \\
X_{j, N_{\tau}^{(3)}}^{(3)} \text { otherwise }
\end{array}\right.
$$

- $I_{0}^{(4)}=\left(I_{1,0}^{(4)}, \ldots, I_{n, 0}^{(4)}\right)$ where

$$
I_{j, 0}^{(4)}=\left\{\begin{array}{l}
0 \text { if } 1 \leq j \leq N_{c}^{\zeta} \\
0 \text { if } N_{p}^{\zeta}>0 \text { and } j \in \bar{\chi}_{\zeta} \\
I_{j, N_{\tau}^{(3)}}^{(3)} \text { otherwise }
\end{array}\right.
$$

- $S_{0}^{(4)}=\left(S_{N_{\tau}^{(3)}}^{(3)}+K_{\zeta-\tau}^{(1)}\right) \mathbf{1}_{\left\{-S_{N_{\tau}^{(3)}}^{(3)} \geq K_{\zeta-\tau}^{(1)}\right\}}$ : if the number of unavailable components is upper than the spare parts arrivals then there are still unavailable components;

- $D_{0}^{(4)}=\left(D_{2, N_{\tau}^{(3)}}^{(3)}, \ldots, D_{m, N_{\tau}^{(3)}}^{(3)}\right): \quad D_{1, N_{\tau}^{(3)}}^{(3)}=\zeta \quad$ is removed;

- $L_{0}^{(4)}=K_{\zeta}^{(3)}-N_{p}^{\zeta} \times \mathbf{1}_{\left\{N_{p}^{\zeta}>0\right\}}$ : the number of deferred PR is equal to the number of components awaiting a PR decreased by the number of remaining spare parts;

- The cost of CR, of PR and of downtime of components are added to the cumulated cost:

$$
\begin{aligned}
C_{0}^{(4)} & =C_{N_{\tau}^{(3)}}^{(3)}+K_{\zeta-\tau}^{(1)} \times c_{s}^{p} \times e^{-\alpha \zeta}+C_{u n a}^{\zeta} \\
& +N_{c}^{\zeta} \times c_{c} \times e^{-\alpha \zeta} \\
& +N_{p}^{\zeta} \times c_{p} \times e^{-\alpha \zeta} \times \mathbf{1}_{\left\{N_{p}^{\zeta}>0\right\}}
\end{aligned}
$$

where

$$
C_{\text {una }}^{\zeta}=\left(C_{\text {una }} / \alpha\right) \times \sum_{j=1}^{N_{c}^{\zeta}}\left(e^{-\alpha X_{j, N_{\tau}^{(3)}}^{(3)}}-e^{-\alpha \zeta}\right)
$$

In this case, after the initialization of Markov chain $Z^{(4)}$, the transitions of $Z^{(4)}$ differ from $Z^{(1)}$ at the 
delivery of a spare part. If there is deferred PR at the arrival time of a spare part, a PR takes place if all components are working. Else, the new spare part supplies the stock. At a failure time, a new spare part is ordered if the failed component has already been replaced. The transition function of $Z^{(4)}$ is defined by:

$$
\begin{aligned}
Z_{k+1}^{(4)} & =\phi_{4}\left(Z_{k}^{(4)}, U_{k+1}\right) \\
& = \begin{cases}\phi_{1}\left(Z_{k}^{(4)}, U_{k+1}(1)\right) & \text { if no deferred PR } \\
\phi_{4,1}\left(Z_{k}^{(4)}, U_{k+1}\right) & \text { otherwise }\end{cases}
\end{aligned}
$$

The transition function of Markov chain $Z^{(4)}$ when $\mathrm{PR}$ are deferred and $t \in(\zeta, H-\tau)$ is now presented. Under PM strategy, if the postponed PR are pending, this reflects that the stock is empty. Thus, the transition function $\phi_{4,1}$ is only presented when the stock is empty and $t \in(\zeta, H-\tau)$.

Firstly, a failure occurs before a spare part delivery. If the failed component has already been replaced (CR or PR), an order is performed. In the opposite case, the $\mathrm{PR}$ of failed component is cancelled and the number of deferred PR is decreased by 1 .

$$
\begin{aligned}
& \phi_{4,1}(((x, i), s, d, c, l, t), u) \\
& =\left((\bar{x}, \bar{i}), s-1, d, c, l-1, x_{1-s}\right) \mathbf{1}_{\left\{i_{1-s}=1\right\}} \\
& +\left((x, i), s-1, \bar{d}, c+c_{s}^{c} \times e^{-\alpha x_{1-s}}, l, x_{1-s}\right) \mathbf{1}_{\left\{i_{1-s}=0\right\}}
\end{aligned}
$$

where

$$
\begin{aligned}
& (\bar{x}, \bar{i})=\left(\left(x_{1}, i_{1}\right), \ldots,\left(x_{1-s}, 0\right), \ldots,\left(x_{n}, i_{n}\right)\right), \\
& \bar{d}=\left(d, x_{1-s}+\tau\right) .
\end{aligned}
$$

Secondly, a spare part is delivered before a failure of component. If the unavailable components are pending then the oldest unavailable component is repaired, else a PR can be performed and the component which will be replaced preventively is randomly chosen with the same probability among components awaiting a PR.

$$
\begin{aligned}
& \phi_{4,1}(((x, i), s, d, c, l, t), u) \\
& =\left(G(\hat{x}, \hat{i}), 0,\left(d_{2}, \ldots, d_{m}\right), c+c_{p} e^{-\alpha d_{1}}, l-1, d_{1}\right) \mathbf{1}_{\{s=0\}} \\
& +\left(G(\tilde{x}, \tilde{i}), s+1,\left(d_{2}, \ldots, d_{m}\right), c+\bar{C}, l, d_{1}\right) \mathbf{1}_{\{s<0\}}
\end{aligned}
$$

where

$$
\begin{aligned}
& \bar{C}=c_{c} \times e^{-\alpha d_{1}}+\left(C_{u n a} / \alpha\right) \times\left(e^{-\alpha x_{1}}-e^{-\alpha d_{1}}\right), \\
& (\tilde{x}, \tilde{i})=\left(\left(d_{1}+F^{-1}\left(u_{1}\right), 0\right),\left(x_{2}, i_{2}\right), \ldots,\left(x_{n}, i_{n}\right)\right),
\end{aligned}
$$

$$
\begin{aligned}
& (\hat{x}, \hat{i})=\left(\left(\hat{x}_{1}, \hat{i}_{1}\right), \ldots,\left(\hat{x}_{n}, \hat{i}_{n}\right)\right) \text { with } \\
& \left(\hat{x}_{j}, \hat{i}_{j}\right)=\left(x_{j}+F^{-1}\left(u_{1}\right) \times \mathbf{1}_{\left\{u_{2} \in A_{j}\right\}}, i_{j} \times \mathbf{1}_{\left\{u_{2} \notin A_{j}\right\}}\right)
\end{aligned}
$$$$
\text { for } j=\{1, \ldots, n\}
$$

and

$$
A_{v}=\left[\frac{\sum_{j=1}^{v-1} i_{j}}{\sum_{j=1}^{n} i_{j}}, \frac{\sum_{j=1}^{v} i_{j}}{\sum_{j=1}^{n} i_{j}}\right) .
$$

For $t \in[H-\tau, H]$, no order is performed. Then, when a component fails and the stock is empty, it is unavailable up to time $H$.

As the CM strategy at the operation horizon term $H$, the cost of downtime of failed components is added and the cumulated discounted cost up to $H$ for PM strategy is:

$$
C_{H}^{(2)}=C_{N_{H-\zeta}^{(4)}}^{(4)}+C_{u n a}^{H}
$$

with

$$
\begin{aligned}
C_{\text {una }}^{H} & =C_{\text {una }} \sum_{j=1}^{n}\left(\int_{X_{j, N_{H-\zeta}^{(4)}}^{H}}^{H} e^{-\alpha t} d t\right) \mathbf{1}_{\left\{X_{j, N_{H-\zeta}^{(4)}}^{(4)} \leq H\right\}} \\
& =\frac{C_{\text {una }}}{\alpha} \sum_{j=1}^{n}\left(e^{-\alpha X_{j, N}^{(4)}(4)}-e^{-\alpha H}\right) \mathbf{1}_{\left\{X_{j, N_{H-\zeta}^{(4)}}^{(4)} \leq H\right\}} \leq
\end{aligned}
$$

$C_{H}^{(2)}$ is the cumulated discounted cost at time $H$ of the PM strategy and $Z_{N_{H-\zeta}^{(4)}}^{(4)}$ is the state of the Markov chain $Z^{(4)}$ just before $H$. 